\title{
Multi-line Herschel/HIFI observations of water reveal infall motions and chemical segregation around high-mass protostars $\star, \star \star$
}

\author{
F. F. S. van der Tak ${ }^{1,2}$, R. F. Shipman ${ }^{1,2}$, T. Jacq ${ }^{3}$, F. Herpin ${ }^{3}$, J. Braine ${ }^{3}$, and F. Wyrowski ${ }^{4}$ \\ ${ }^{1}$ SRON Netherlands Institute for Space Research, Landleven 12, 9747 AD Groningen, The Netherlands \\ e-mail: vdtak@sron.nl \\ ${ }^{2}$ Kapteyn Astronomical Institute, University of Groningen, Groningen, The Netherlands \\ ${ }^{3}$ Université de Bordeaux, Bordeaux, France \\ ${ }^{4}$ Max-Planck-Institut für Radioastronomie, Auf dem Hügel 69, 53121 Bonn, Germany
}

Received 6 July 2018 / Accepted 18 March 2019

\begin{abstract}
Context. The physical conditions during high-mass star formation are poorly understood. Outflow and infall motions have been detected around massive protostellar objects, but their dependence on mass, luminosity, and age is unclear. In addition, physical conditions and molecular abundances are often estimated using simple assumptions such as spherical shape and chemical homogeneity, which may limit the accuracy of the results.

Aims. We aim to characterize the dust and gas distribution and kinematics of the envelopes of high-mass protostars. In particular, we search for infall motions, abundance variations, and deviations from spherical symmetry, using Herschel data from the WISH program. Methods. We used HIFI maps of the $987 \mathrm{GHz} \mathrm{H_{2 }} \mathrm{O}_{202}-1_{11}$ emission to measure the sizes and shapes of 19 high-mass protostellar envelopes. To identify infall, we used HIFI spectra of the optically thin $\mathrm{C}^{18} \mathrm{O} 9-8$ and $\mathrm{H}_{2}^{18} \mathrm{O} 1_{11}-0_{00}$ lines. The high- $J \mathrm{C}^{18} \mathrm{O}$ line traces the warm central material and redshifted $\mathrm{H}_{2}^{18} \mathrm{O} 1_{11}-0_{00}$ absorption indicates material falling onto the warm core. We probe small-scale chemical differentiation by comparing $\mathrm{H}_{2} \mathrm{O} 752$ and $987 \mathrm{GHz}$ spectra with those of $\mathrm{H}_{2}^{18} \mathrm{O}$.

Results. Our measured radii of the central part of the $\mathrm{H}_{2} \mathrm{O} 22_{02}-1_{11}$ emission are $30-40 \%$ larger than the predictions from spherical envelope models, and axis ratios are $<2$, which we consider good agreement. For 11 of the 19 sources, we find a significant redshift of the $\mathrm{H}_{2}^{18} \mathrm{O} 1_{11}-0_{00}$ line relative to $\mathrm{C}^{18} \mathrm{O} 9-8$. The inferred infall velocities are $0.6-3.2 \mathrm{~km} \mathrm{~s}^{-1}$, and estimated mass inflow rates range from $7 \times 10^{-5}$ to $2 \times 10^{-2} M_{\odot} \mathrm{yr}^{-1}$. The highest mass inflow rates seem to occur toward the sources with the highest masses, and possibly the youngest ages. The other sources show either expanding motions or $\mathrm{H}_{2}^{18} \mathrm{O}$ lines in emission. The $\mathrm{H}_{2}^{18} \mathrm{O} 1_{11}-0_{00}$ line profiles are remarkably similar to the differences between the $\mathrm{H}_{2} \mathrm{O} 22_{02}-1_{11}$ and $2_{11}-2_{02}$ profiles, suggesting that the $\mathrm{H}_{2}^{18} \mathrm{O}$ line and the $\mathrm{H}_{2} \mathrm{O}$ $2_{02}-1_{11}$ absorption originate just inside the radius where water evaporates from grains, typically 1000-5000 au from the center. In some sources, the $\mathrm{H}_{2}^{18} \mathrm{O}$ line is detectable in the outflow, where no $\mathrm{C}^{18} \mathrm{O}$ emission is seen.

Conclusions. Together, the $\mathrm{H}_{2}^{18} \mathrm{O}$ absorption and $\mathrm{C}^{18} \mathrm{O}$ emission profiles show that the water abundance around high-mass protostars has at least three levels: low in the cool outer envelope, high within the $100 \mathrm{~K}$ radius, and very high in the outflowing gas. Thus, despite the small regions, the combination of lines presented in this work reveals systematic inflows and chemical information about the outflows.
\end{abstract}

Key words. stars: formation - ISM: molecules - astrochemistry

\section{Introduction}

High-mass stars $\left(>8 M_{\odot}\right)$ play a key role in the evolution of their host galaxies, but their formation is poorly understood, especially for masses $>20 M_{\odot}$. The leading models of high-mass star formation involve infall from a dense protostellar core, and accretion onto the protostar via a circumstellar disk (Tan et al. 2014; Motte et al. 2018). While rotating disks have been detected around young B-type (Sánchez-Monge et al. 2013; Beltrán \& de Wit 2016) and O-type (Johnston et al. 2015; Cesaroni et al.

\footnotetext{
* Copies of the maps and reduced spectra (FITS files) are available at the CDS via anonymous ftp to cdsarc.u-strasbg.fr (130.79.128.5) or via http://cdsarc.u-strasbg.fr/viz-bin/ qcat? J/A+A/625/A103

${ }^{\star \star}$ Herschel is an ESA space observatory with science instruments provided by European-led Principal Investigator consortia and with important participation from NASA.
}

2017) protostars, the exact manner in (and rate at) which material is gathered from the surroundings is still a matter of debate.

In the "monolithic collapse" model, a massive dense core collapses under its own gravity and forms a (cluster of) protostar(s), much like the low-mass case. This picture is supported by observations of massive collimated outflows from high-mass protostars (Beuther et al. 2002). In the alternative "competitive accretion" model, the accreting protostellar core is replenished from the surroundings. Evidence supporting this model comes, for example, from observations of extended contracting motions in pre-protocluster regions (Pillai et al. 2011). It is possible that both models are valid under different conditions, or that combination models need to be developed (Peters et al. 2011). To constrain such models, observations of suitable tracers are essential.

Large-scale $(\sim 0.1 \mathrm{pc})$ infall motions have been detected toward high-mass star-forming regions in ground-based 
submillimeter-wave molecular emission line maps (Motte et al. 2003; Peretto et al. 2006), in redshifted $\mathrm{NH}_{3}$ line absorption at centimeter wavelengths (Sollins et al. 2005; Beltrán et al. 2006), and recently in SOFIA $\mathrm{NH}_{3}$ spectra (Wyrowski et al. 2012, 2016). Searches for infall in unbiased selections from catalogs of high-mass star-forming regions confirm the ubiquity of such motions (Fuller et al. 2005; Klaassen \& Wilson 2007; He et al. 2015; Cunningham et al. 2018).

The water molecule appears to be a promising tracer of infall motions in low-mass star-forming regions (Mottram et al. 2013), and San José-García et al. (2016) linked water observations between low- and high-mass star-forming regions. Spectra of low- $J$ line emission toward high-mass objects often exhibit inverse P Cygni profiles (Van der Tak et al. 2013), which have been modeled successfully as infall, using spherical Monte Carlo models (Herpin et al. 2016). Stronger evidence comes from maps of the luminous mini-starburst region W43 in low-energy $\mathrm{H}_{2} \mathrm{O}$ and $\mathrm{H}_{2}^{18} \mathrm{O}$ lines (Jacq et al. 2016): extended $\mathrm{H}_{2}^{18} \mathrm{O}$ absorption that is redshifted with respect to the ${ }^{13} \mathrm{CO} 10-9$ emission clearly indicates infall motions.

The $\mathrm{H}_{2}^{18} \mathrm{O} 1_{11}-0_{00}$ ground-state absorption toward W43MM1 is remarkable because its shape closely matches that of the central absorption feature in the $\mathrm{H}_{2} \mathrm{O} 2_{02}-1_{11}$ excited-state emission line (Jacq et al. 2016). This resemblance strongly suggests that the two lines originate in the same gas, which is curious given their different excitation energies (101 vs. $0 \mathrm{~K}$ ). In order to understand the similarity of these line profiles, this paper explores whether the same effect is seen in other high-mass protostars.

Another puzzle in previous observations of $\mathrm{H}_{2} \mathrm{O}$ lines toward high-mass protostars concerns their line shape (Van der Tak et al. 2013). The profiles show narrow line cores from the protostellar envelopes, and broad line wings from the outflows, but the wings are much more pronounced at redshifted than at blueshifted velocities, and often the blueshifted wings are nearly or entirely missing from the profiles. This asymmetry cannot be due to continuum absorption (e.g. by a disk) which would preferentially affect background gas (i.e., receding velocities). Special geometrical configurations may explain individual cases, but not a sample of many sources. One possibility is that the $\mathrm{H}_{2} \mathrm{O}$ excitation temperature is close to the brightness temperature of the background, so that no net line emission or absorption appears in the spectra. To explore the origin of the asymmetry, this paper explores its dependence on line properties such as excitation energy and critical density.

This paper uses multi-line maps and spectra of $\mathrm{H}_{2} \mathrm{O}$ and $\mathrm{H}_{2}^{18} \mathrm{O}$ lines toward a sample of high-mass protostars to explore their gas distribution and dynamics. In particular, we compare $\mathrm{H}_{2}^{18} \mathrm{O}$ line profiles to those of $\mathrm{C}^{18} \mathrm{O}$ to search for velocity shifts due to infall motions. Furthermore, we use $\mathrm{H}_{2} \mathrm{O}$ maps to measure the sizes of the protostellar envelopes, and to test the assumption of spherical symmetry in previous analyses of pointed spectra. Section 2 describes our observations, and Sect. 3 presents the resulting maps and spectra. Section 4 compares our derived infall rates with previous observations and with models, and searches for trends with basic source parameters. Finally, Sect. 5 describes our conclusions.

\section{Observations}

\subsection{Source sample}

As part of the guaranteed time program WISH (Water In Starforming regions with Herschel; Van Dishoeck et al. 2011), we have selected 19 regions of high-mass star formation for observation in lines of $\mathrm{H}_{2} \mathrm{O}$ and its isotopes with the Heterodyne Instrument for the Far Infrared (HIFI; De Graauw et al. 2010) on ESA's Herschel Space Observatory (Pilbratt et al. 2010). The sources were selected to cover wide ranges in bolometric luminosity, mid-infrared brightness, and circumstellar mass, and to include regions with hot molecular cores and ultracompact HII regions; see Van der Tak et al. (2013) for details. Table 1 presents the source sample, where distances are updated following König et al. (2017), and luminosities and masses are scaled assuming a simple $d^{2}$ dependence.

Most of the updated distances are direct determinations using trigonometric maser parallax observations. The near kinematic distance for G327 seems to be broadly accepted in the recent literature. Only the case of G31.41 is more complicated. The commonly used distance for G31.41 is $7.9 \mathrm{kpc}$, based on its radial velocity from the Sun and position on the sky, coupled with a Galactic rotation model (Churchwell et al. 1990). However, such kinematically derived distances can be off by factors of $\gtrsim 2$ in either direction; AFGL 2591 and W33A are cases in point (Rygl et al. 2012; Immer et al. 2013). Alternatively, G31.41 may be associated with the W43-Main cloud complex, as suggested by position-velocity diagrams of the molecular gas in the surroundings (Nguyen Luong et al. 2011). For W43-Main, two distance estimates exist that are based on Very Long Baseline Interferometry (VLBI) observations of maser parallax (see also Beltrán et al. 2018). Reid et al. (2014) reported a distance of $4.9 \mathrm{kpc}$ to the W43-Main core, while Zhang et al. (2014) reported distances to five maser spots with distances ranging from 6.21 to $4.27 \mathrm{kpc}$. Given this large spread, we adopt a distance of $4.9 \mathrm{kpc}$ for G31.41 in this paper, and recommend a specific maser parallax study of G31.41 itself.

\subsection{Data acquisition and reduction}

Maps of the $\mathrm{H}_{2} \mathrm{O} \quad 2_{02}-1_{11}$ line at $987.927 \mathrm{GHz}$ (hereafter $987 \mathrm{GHz}$ ) were taken with HIFI band $4 \mathrm{a}$. The maps are $1^{\prime}$ on the side, and were taken in on-the-fly (OTF) observing mode. The backend was the acousto-optical Wide-Band Spectrometer (WBS) which provides a bandwidth of $4 \times 1140 \mathrm{MHz}$ $\left(1200 \mathrm{~km} \mathrm{~s}^{-1}\right)$ at a resolution of $1.1 \mathrm{MHz}\left(0.3 \mathrm{~km} \mathrm{~s}^{-1}\right)$. Table 2 presents a detailed observation log including integration times; system temperatures were around $340 \mathrm{~K}$. The FWHM beam size at this frequency is 22" (Roelfsema et al. 2012), which corresponds to $0.14-0.92 \mathrm{pc}$ at the distances of our sources. The maps thus cover at least part of the protostellar outflows, while the beam resolves the protostellar envelopes, but not any possible disks.

Spectra of the $\mathrm{H}_{2}^{18} \mathrm{O} 1_{11}-0_{00}$ line at $1101.698 \mathrm{GHz}$ (hereafter $1101 \mathrm{GHz}$ ), the $\mathrm{H}_{2} \mathrm{O} 2_{11}-2_{02}$ line at $752.033 \mathrm{GHz}$ (hereafter $752 \mathrm{GHz}$ ), the ${ }^{13} \mathrm{CO} 10-9$ line at $1101.34976 \mathrm{GHz}$, and the $\mathrm{C}^{18} \mathrm{O}$ 9-8 line at $987.560 \mathrm{GHz}$ were obtained toward the same sources with HIFI, using the Double Beam Switch (DBS) observing mode with a chopper throw of $3^{\prime}$. The $\mathrm{C}^{18} \mathrm{O}$ and ${ }^{13} \mathrm{CO}$ lines were observed in the same tuning as the $\mathrm{H}_{2} \mathrm{O} 2_{11}-2_{02}$ and the $\mathrm{H}_{2}^{18} \mathrm{O}$ $1_{11}-0_{00}$ lines, respectively, and thus share the same ObsIDs. Table 2 lists the integration times of the spectra; system temperatures were around 200 and $390 \mathrm{~K}$ for the $752 \mathrm{GHz}$ and $\sim 1 \mathrm{THz}$ lines, respectively. The pointed 987 and $752 \mathrm{GHz}$ spectra have been presented before by San José-García et al. (2016); the ${ }^{13} \mathrm{CO}$ and $C^{18} \mathrm{O}$ spectra were presented in San José-García et al. (2013). The DBS spectra have higher noise per second of integration than the maps at the same frequency, which represents the noise penalty to be paid for stabilizing the system by differencing two 
Table 1. Source sample.

\begin{tabular}{|c|c|c|c|c|c|c|}
\hline Source ${ }^{(a)}$ & $\begin{array}{l}\text { RA (J2000.0) } \\
\text { hh mm ss.s }\end{array}$ & $\begin{array}{l}\text { Dec } \\
0,1,\end{array}$ & $\begin{array}{c}L_{\mathrm{bol}} \\
L_{\odot}\end{array}$ & $\begin{array}{c}d \\
\mathrm{kpc}\end{array}$ & $\begin{array}{c}M_{\mathrm{env}} \\
M_{\odot}\end{array}$ & $\begin{array}{l}\text { Distance } \\
\text { reference }\end{array}$ \\
\hline \multicolumn{7}{|c|}{ Mid-IR-quiet HMPOs ${ }^{(b)}$} \\
\hline IRAS $05358+3543$ & 053913.1 & +354550 & $6.3 \times 10^{3}$ & 1.8 & 142 & (1) \\
\hline IRAS 16272-4837 & 163058.7 & -484355 & $2.4 \times 10^{4}$ & 3.4 & 2170 & (1) \\
\hline NGC 6334I(N) & 172055.2 & -354504 & $1.1 \times 10^{3}$ & 1.3 & 2237 & (5) \\
\hline W43 MM1 & 184747.0 & -015428 & $1.8 \times 10^{4}$ & 5.5 & 5992 & (2) \\
\hline $\mathrm{DR} 21(\mathrm{OH})$ & 203900.8 & +422248 & $1.3 \times 10^{4}$ & 1.5 & 472 & (1) \\
\hline \multicolumn{7}{|l|}{ Mid-IR-bright HMPOs } \\
\hline W3 IRS5 & 022540.6 & +620551 & $1.7 \times 10^{5}$ & 2.0 & 424 & (1) \\
\hline IRAS 18089-1732 & 181151.5 & -173129 & $1.3 \times 10^{4}$ & 2.3 & 172 & (1) \\
\hline W33A & 181439.1 & -175207 & $4.4 \times 10^{4}$ & 2.4 & 700 & (1) \\
\hline IRAS 18151-1208 & 181758.0 & -120727 & $2.0 \times 10^{4}$ & 2.9 & 153 & (1) \\
\hline AFGL 2591 & 202924.7 & +401119 & $2.2 \times 10^{5}$ & 3.3 & 363 & (1) \\
\hline \multicolumn{7}{|l|}{ Hot molecular cores } \\
\hline G327-0.6 & 155308.8 & -543701 & $4.4 \times 10^{4}$ & 3.1 & 1804 & (6) \\
\hline NGC 6334I & 172053.3 & -354700 & $1.5 \times 10^{5}$ & 1.3 & 439 & (5) \\
\hline G29.96-0.02 & 184603.8 & -023922 & $2.7 \times 10^{5}$ & 5.3 & 599 & (2) \\
\hline G31.41+0.31 & 184734.3 & -011246 & $8.8 \times 10^{4}$ & 4.9 & 1142 & (2) \\
\hline \multicolumn{7}{|c|}{ Ultracompact HII regions } \\
\hline G5.89-0.39 (W28A) & 180030.4 & -240402 & $5.1 \times 10^{4}$ & 1.3 & 140 & (1) \\
\hline $\mathrm{G} 10.47+0.03$ & 180838.2 & -195150 & $8.1 \times 10^{5}$ & 8.6 & 2568 & (3) \\
\hline G34.26+0.15 & 185318.6 & +011458 & $7.5 \times 10^{4}$ & 1.6 & 421 & (4) \\
\hline W51N-e1 & 192343.8 & +143026 & $1.1 \times 10^{5}$ & 5.4 & 5079 & (7) \\
\hline NGC 7538-IRS1 & 231345.3 & +612810 & $1.3 \times 10^{5}$ & 2.7 & 433 & (1) \\
\hline
\end{tabular}

Notes. ${ }^{(a)}$ The text uses "short" source names, which is the part preceding the + or - sign. ${ }^{(b)}$ High-Mass Protostellar Objects.

References. (1) Van der Tak et al. (2013); (2) Zhang et al. (2014); (3) Sanna et al. (2014); (4) Kurayama et al. (2011); Xu et al. (2016); (5) Wu et al. (2014); (6) Wienen et al. (2015); (7) Sato et al. (2010).

reference positions in the DBS observing mode. For the $\mathrm{H}_{2}^{18} \mathrm{O}$ and $\mathrm{C}^{18} \mathrm{O}$ lines, the beam size of $20-22^{\prime \prime}$ is very similar to that of the $987 \mathrm{GHz}$ maps, which permits a direct comparison of the results. The beam size of the $752 \mathrm{GHz}$ observations is $28^{\prime \prime}$.

The data are Herschel/HIFI standard products (Shipman et al. 2017) with further processing performed in the Herschel Interactive Processing Environment (HIPE; Ott 2010) version 15; further analysis was carried out in the CLASS ${ }^{1}$ package, version of December 2015 or later. Raw antenna temperatures were converted to $T_{\mathrm{mb}}$ scale using a main beam efficiency of $63 \%$ for both frequencies around $1 \mathrm{THz}$ and $64 \%$ for the $752 \mathrm{GHz}$ line $^{2}$, and linear baselines were subtracted. After inspection, the data from the two polarization channels were averaged to obtain the rms noise levels reported in Table 2. The absolute calibration uncertainty of HIFI bands 3 and 4 is estimated to be $10-15 \%$, but the relative calibration between lines in the same spectrum should be much better, which is relevant for $\mathrm{C}^{18} \mathrm{O}$ and ${ }^{13} \mathrm{CO}$.

\section{Results}

\subsection{Line profiles of $\mathrm{H}_{2}^{18} \mathrm{O},{ }^{13} \mathrm{CO}$, and $\mathrm{C}^{18} \mathrm{O}$}

Figure 1 shows the observed velocity profiles of the $\mathrm{H}_{2}^{18} \mathrm{O} 1_{11}-0_{00}$ and $\mathrm{C}^{18} \mathrm{O} 9-8$ lines. For IRAS 18151, we show

\footnotetext{
1 http://wwW.iram.fr/IRAMFR/GILDAS

2 https://www. cosmos.esa.int/web/herschel/

legacy-documentation-hifi-level-2
}

the $\mathrm{H}_{2} \mathrm{O} 1_{11}-0_{00}$ line as the $\mathrm{H}_{2}^{18} \mathrm{O}$ line is not detected. For IRAS 05358, IRAS 16272, and IRAS 18151, the $\mathrm{C}^{18} \mathrm{O}$ 9-8 line is weak, so we use the ${ }^{13} \mathrm{CO} 10-9$ line to measure velocities. For the other sources, the data indicate substantial optical depth in the ${ }^{13} \mathrm{CO} 10-9$ line, so we prefer $\mathrm{C}^{18} \mathrm{O} 9-8$ as velocity standard.

While the $\mathrm{C}^{18} \mathrm{O}$ (or ${ }^{13} \mathrm{CO}$ ) lines appear purely in emission for all sources, the $\mathrm{H}_{2}^{18} \mathrm{O}$ (or $\mathrm{H}_{2} \mathrm{O}$ ) profiles show absorption, in some cases mixed with emission. Despite this difference, the peak of the $\mathrm{H}_{2}^{18} \mathrm{O}$ absorption is seen to lie close to the peak of the $\mathrm{C}^{18} \mathrm{O}$ (or ${ }^{13} \mathrm{CO}$ ) emission, but at a measurable velocity offset. In most cases, the $\mathrm{H}_{2}^{18} \mathrm{O}$ absorption peak is significantly redshifted from the $\mathrm{C}^{18} \mathrm{O}$ (or ${ }^{13} \mathrm{CO}$ ) emission peak, by $0.6-3.2 \mathrm{~km} \mathrm{~s}^{-1}$. Table 3 reports the peak velocities of the $\mathrm{H}_{2}^{18} \mathrm{O}$ absorption and $\mathrm{C}^{18} \mathrm{O}$ (or ${ }^{13} \mathrm{CO}$ ) emission, as estimated directly from the HIFI spectra. We estimate the uncertainty on these velocities to be $\approx 0.3 \mathrm{~km} \mathrm{~s}^{-1}$. In some cases, no blueshifted absorption or no absorption at all is seen.

The $\mathrm{C}^{18} \mathrm{O} 9-8$ line has a relatively high upper level energy $(237 \mathrm{~K})$ and critical density $\left(7.7 \times 10^{5} \mathrm{~cm}^{-3}\right)$, using spectroscopy from Endres et al. (2016) and collision data from Yang et al. (2010), as provided on the Leiden Atomic and Molecular Database (LAMDA; Schöier et al. 2005). Since in addition the $\mathrm{C}^{18} \mathrm{O}$ abundance is likely to be low $\left(\sim 10^{-6}\right)$, this line should be an optically thin tracer of the warm dense gas close to the central protostar. For the three sources with weak $\mathrm{C}^{18} \mathrm{O}$ 9-8 emission, this argument also seems to hold for the 
Table 2. Observation log for the $\mathrm{H}_{2}^{18} \mathrm{O}$ pointed observations and $\mathrm{H}_{2} \mathrm{O} 987 \mathrm{GHz}$ maps.

\begin{tabular}{|c|c|c|c|c|}
\hline Source & Species & $\operatorname{ObsID}^{(a)}$ & $\begin{array}{c}t_{\text {int }}{ }^{(b)} \\
(\mathrm{s})\end{array}$ & $\begin{array}{r}\operatorname{rms}^{(c)} \\
(\mathrm{mK})\end{array}$ \\
\hline \multirow[t]{2}{*}{ IRAS 05358} & $\mathrm{H}_{2}^{18} \mathrm{O}$ & 206124,206126 & 3566 & 20 \\
\hline & $\mathrm{H}_{2} \mathrm{O}$ & 204508 & 5.86 & 481 \\
\hline \multirow[t]{2}{*}{ IRAS 16272} & $\mathrm{H}_{2}^{18} \mathrm{O}$ & 214417,214419 & 3455 & 20 \\
\hline & $\mathrm{H}_{2} \mathrm{O}$ & 203166 & 5.86 & 473 \\
\hline \multirow[t]{2}{*}{ NGC 6334I(N) } & $\mathrm{H}_{2}^{18} \mathrm{O}$ & 206383 & 2965 & 22 \\
\hline & $\mathrm{H}_{2} \mathrm{O}$ & 204523 & 5.86 & 481 \\
\hline \multirow[t]{2}{*}{ W43-MM1 } & $\mathrm{H}_{2}^{18} \mathrm{O}$ & 191670,207372 & 3566 & 20 \\
\hline & $\mathrm{H}_{2} \mathrm{O}$ & 215899 & 5.86 & 524 \\
\hline \multirow[t]{2}{*}{$\mathrm{DR} 21(\mathrm{OH})$} & $\mathrm{H}_{2}^{18} \mathrm{O}$ & 194794, 197974 & 3566 & 20 \\
\hline & $\mathrm{H}_{2} \mathrm{O}$ & 210042 & 7.86 & 396 \\
\hline \multirow[t]{2}{*}{ W3IRS5 } & $\mathrm{H}_{2}^{18} \mathrm{O}$ & 191658,201591 & 3566 & 20 \\
\hline & $\mathrm{H}_{2} \mathrm{O}$ & 203160 & 5.86 & 498 \\
\hline \multirow[t]{2}{*}{ IRAS 18089} & $\mathrm{H}_{2}^{18} \mathrm{O}$ & 229882,229883 & 3455 & 20 \\
\hline & $\mathrm{H}_{2} \mathrm{O}$ & 218210 & 5.86 & 557 \\
\hline \multirow[t]{2}{*}{ W33A } & $\mathrm{H}_{2}^{18} \mathrm{O}$ & 191638,208086 & 3566 & 20 \\
\hline & $\mathrm{H}_{2} \mathrm{O}$ & 215902 & 5.86 & 465 \\
\hline \multirow[t]{2}{*}{ IRAS 18151} & $\mathrm{H}_{2}^{18} \mathrm{O}$ & 229880,229881 & 3455 & 20 \\
\hline & $\mathrm{H}_{2} \mathrm{O}$ & 218212 & 5.86 & 659 \\
\hline \multirow[t]{2}{*}{ AFGL 2591} & $\mathrm{H}_{2}^{18} \mathrm{O}$ & 194795,197973 & 3566 & 20 \\
\hline & $\mathrm{H}_{2} \mathrm{O}$ & 210038 & 5.86 & 446 \\
\hline \multirow[t]{2}{*}{ G327 } & $\mathrm{H}_{2}^{18} \mathrm{O}$ & $214422,214423,214425,214426$ & 3428 & 21 \\
\hline & $\mathrm{H}_{2} \mathrm{O}$ & 203169 & 5.86 & 490 \\
\hline \multirow[t]{2}{*}{ NGC 6334I } & $\mathrm{H}_{2}^{18} \mathrm{O}$ & 206385 & 2965 & 22 \\
\hline & $\mathrm{H}_{2} \mathrm{O}$ & 204522 & 5.86 & 486 \\
\hline \multirow[t]{2}{*}{ G29.96 } & $\mathrm{H}_{2}^{18} \mathrm{O}$ & $191668,191669,229875,229876$ & 3700 & 20 \\
\hline & $\mathrm{H}_{2} \mathrm{O}$ & 207655 & 5.86 & 475 \\
\hline \multirow[t]{2}{*}{ G31.41 } & $\mathrm{H}_{2}^{18} \mathrm{O}$ & $191671,191672,229873,229874$ & 3700 & 20 \\
\hline & $\mathrm{H}_{2} \mathrm{O}$ & 207654 & 5.86 & 477 \\
\hline \multirow[t]{2}{*}{ G5.89 } & $\mathrm{H}_{2}^{18} \mathrm{O}$ & 229888, 229889, 229890, 229891 & 3148 & 21 \\
\hline & $\mathrm{H}_{2} \mathrm{O}$ & 218201 & 5.86 & 575 \\
\hline \multirow[t]{2}{*}{ G10.47 } & $\mathrm{H}_{2}^{18} \mathrm{O}$ & $229884,229885,229886,229887$ & 3148 & 21 \\
\hline & $\mathrm{H}_{2} \mathrm{O}$ & 218208 & 5.86 & 476 \\
\hline \multirow[t]{2}{*}{ G34.26 } & $\mathrm{H}_{2}^{18} \mathrm{O}$ & 191673, 191674, 229871, 229872 & 3700 & 20 \\
\hline & $\mathrm{H}_{2} \mathrm{O}$ & 207652 & 5.86 & 493 \\
\hline \multirow[t]{2}{*}{ W51 } & $\mathrm{H}_{2}^{18} \mathrm{O}$ & 194801, 194802, 207384, 207385 & 3420 & 20 \\
\hline & $\mathrm{H}_{2} \mathrm{O}$ & 207651 & 5.86 & 485 \\
\hline \multirow[t]{2}{*}{ NGC 7538IRS1 } & $\mathrm{H}_{2}^{18} \mathrm{O}$ & $191663,191664,197976$ & 3569 & 20 \\
\hline & $\mathrm{H}_{2} \mathrm{O}$ & 203161 & 5.86 & 479 \\
\hline
\end{tabular}

Notes. ${ }^{(a)}$ The leading 1342 has been omitted. ${ }^{(b)}$ For pointed observations, the integration time is for the total spectra, i.e. all ObsIDs added. For maps, the integration time is per observed position. ${ }^{(c)}$ The rms is the noise in $\delta v=1.1 \mathrm{MHz}$. For pointed observations, the integration time is for the total spectra, i.e. all ObsIDs added.

${ }^{13}$ CO $10-9$ line, presumably owing to a low envelope mass. These three sources are not the lowest luminosity cases in our study, so the low envelope mass and weak $\mathrm{C}^{18} \mathrm{O}$ emission may be an evolutionary effect. The appearance of the $\mathrm{H}_{2}^{18} \mathrm{O}$ absorption at redshifted velocities thus implies infalling motions in the gas surrounding the dense warm cores seen in $\mathrm{C}^{18} \mathrm{O} 9$ 8 and/or ${ }^{13} \mathrm{CO} 10-9$ emission. The velocity difference between the $\mathrm{C}^{18} \mathrm{O}$ and $\mathrm{H}_{2}^{18} \mathrm{O}$ lines indicates approximate infall speeds 

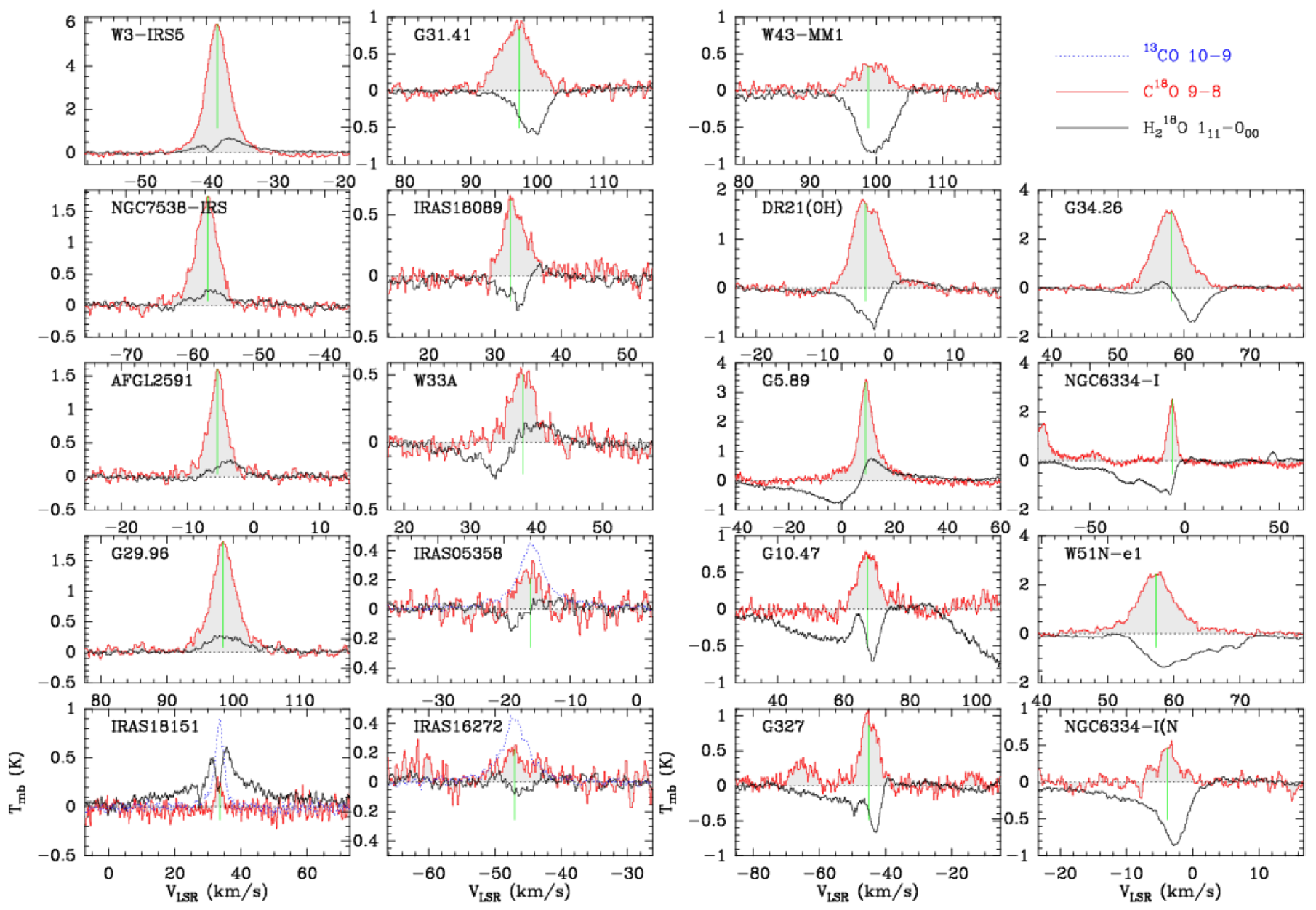

Fig. 1. Line profiles of $\mathrm{H}_{2}^{18} \mathrm{O} 1_{11}-0_{00}$ (black) and $\mathrm{C}^{18} \mathrm{O} 9-8$ (red) toward our 19 sources. The vertical green line denotes the $\mathrm{C}^{18} \mathrm{O}$ velocity in Table 3 . For IRAS 18151, we show $\mathrm{H}_{2} \mathrm{O} 1_{11}-0_{00}$ instead of the $\mathrm{H}_{2}^{18} \mathrm{O}$ line which is not detected. For IRAS 18151, IRAS 16272 and IRAS 05358 , the blue dotted spectrum is ${ }^{13} \mathrm{CO} 10-9$ as $\mathrm{C}^{18} \mathrm{O}$ is weak or noisy. The dip in the G10.47 spectrum at $V_{\mathrm{LSR}}>80 \mathrm{~km} \mathrm{~s}^{-1}$ is an artifact from the image sideband.

between 0.6 and $3.2 \mathrm{~km} \mathrm{~s}^{-1}$, although these values represent line-of-sight averages.

For the sources W3 IRS5, W33A, NGC 6334I, and IRAS 05358, the $\mathrm{H}_{2}^{18} \mathrm{O}$ absorption peak is blueshifted from the $\mathrm{C}^{18} \mathrm{O}$ emission peak, suggesting expanding motions. The line profiles toward G5.89 and G10.47 are complex, and show a mixture of infall and expansion. These two sources are not included in the analysis below.

We emphasize the importance of using a precise velocity standard, in this case the $\mathrm{C}^{18} \mathrm{O} 9-8$ line, for the detection of infall motions. The $\mathrm{C}^{18} \mathrm{O}$ velocities in Table 3 differ from the groundbased values (Van der Tak et al. 2013, Table 1; Van Dishoeck et al. 2011) by up to $1 \mathrm{~km} \mathrm{~s}^{-1}$, which shows that velocity precision is often limited by source inhomogeneities, rather than by spectral resolution or other instrumental parameters.

Wyrowski et al. $(2012,2016)$ have used SOFIA to measure the $\mathrm{NH}_{3} 3_{2}^{+}-2_{2}^{-}$line at $1810.379 \mathrm{GHz}$ toward several of our sources. These authors reported redshifted absorption toward W43 MM1, G327, G31.41, and G34.26, implying infall, and blueshifted absorption toward W33A and G5.89, thereby implying expansion. These results agree qualitatively with ours and their measured velocities are similar to those reported in this work.

Toward G34.26, Hajigholi et al. (2016) have measured infall through multi-line $\mathrm{NH}_{3}$ line observations with HIFI and found two infall components with velocities of 2.7 and $5.3 \mathrm{~km} \mathrm{~s}^{-1}$. The ground-state $\mathrm{H}_{2}^{18} \mathrm{O}$ and $\mathrm{NH}_{3}$ lines presented in this work and by Wyrowski et al. only probe the lower-velocity of these components, which may mean that the higher-velocity component mostly arises in very warm and dense gas in close proximity to the protostar. This result suggests that the infall velocity of the gas increases as it approaches the protostar.

\subsection{Maps of $\mathrm{H}_{2} \mathrm{O}$}

Figures 2 and A.1-A.17 show our maps of the $\mathrm{H}_{2} \mathrm{O} 987 \mathrm{GHz}$ line emission. The greyscale and white contours denote the line core, while the blue and red contours correspond to the blue- and redshifted line wings (see the caption for details). The emission is seen to be compact (except G31.41), mildly elongated, and not to depend much on velocity interval (except NGC 6334I). For IRAS 18151, the emission is too weak to assess its morphology. The map of NGC 7538 is not shown as it suffers from mispointing, so that only limits on the emission size and shape can be obtained.

The observed morphology of the $987 \mathrm{GHz}$ emission does not appear to depend much on velocity interval (Figs. 2 and A.1-A.17). This contrasts with the low- $J$ CO emission from our sources, which shows a clear bipolar morphology, especially at velocities away from line center (see references in Van Dishoeck et al. 2011). We conclude that the bulk of the warm dense gas in the outflow as traced by the $\mathrm{H}_{2} \mathrm{O} 987 \mathrm{GHz}$ line is confined to a 
Table 3. Measured velocities and derived infall rates.

\begin{tabular}{|c|c|c|c|c|}
\hline Source & $\begin{array}{l}V\left(\mathrm{C}^{18} \mathrm{O}\right) \\
\mathrm{km} \mathrm{s}^{-1}\end{array}$ & $\begin{array}{l}V\left(\mathrm{H}_{2}^{18} \mathrm{O}\right) \\
\mathrm{km} \mathrm{s}^{-1}\end{array}$ & $\begin{array}{l}V_{\text {inf }}{ }^{(a)} \\
\mathrm{km} \mathrm{s}^{-1}\end{array}$ & $\begin{array}{c}\dot{M}_{\mathrm{acc}}{ }^{(b)} \\
10^{-3} M_{\odot} \mathrm{yr}^{-1}\end{array}$ \\
\hline IRAS 05358 & $-16.0^{(d)}$ & -18.5 & +2.5 & $-0-$ \\
\hline IRAS 16272 & -47.0 & -46.3 & -0.7 & 1.28 \\
\hline NGC 6334 I(N) & -3.8 & -2.7 & -1.1 & 4.75 \\
\hline W43 MM1 & +98.8 & +99.4 & -0.6 & 6.06 \\
\hline DR21(OH) & -3.5 & -2.2 & -1.3 & 1.86 \\
\hline W3 IRS5 & -38.4 & -39.9 & +1.5 & $-0-$ \\
\hline IRAS 18089 & +32.4 & +33.8 & -1.4 & 3.28 \\
\hline W33A & +38.0 & +33.8 & +4.2 & $-0-$ \\
\hline IRAS 18151 & $+33.6^{(d)}$ & $+33.4^{(e)}$ & -0.2 & 0.07 \\
\hline AFGL $2591^{(c)}$ & -5.5 & $-0-$ & $-0-$ & $-0-$ \\
\hline G327-0.6 & -45.0 & -43.1 & -1.9 & 4.19 \\
\hline NGC 6334I & -6.5 & -7.9 & +1.4 & $-0-$ \\
\hline $\mathrm{G} 29.96^{(c)}$ & +98.5 & $-0-$ & $-0-$ & $-0-$ \\
\hline G31.41 & +97.3 & +99.3 & -2.0 & 9.85 \\
\hline G5.89 $9^{(c)}$ & +9.3 & $-0-$ & $-0-$ & $-0-$ \\
\hline G10.47 & +67.3 & +68.7 & -1.4 & 7.15 \\
\hline G34.26 & +58.0 & +61.2 & -3.2 & 18.9 \\
\hline W51N-e1 & +57.3 & +58.9 & -1.6 & 14.2 \\
\hline NGC $7538^{(c)}$ & -57.6 & $-0-$ & $-0-$ & $-0-$ \\
\hline
\end{tabular}

Notes. ${ }^{(a)} V_{\text {inf }}=V\left(\mathrm{C}^{18} \mathrm{O}\right)-V\left(\mathrm{H}_{2}^{18} \mathrm{O}\right) .{ }^{(b)}$ Zero denotes lack of (blueshifted) absorption w.r.t. the source. ${ }^{(c)} \mathrm{H}_{2}^{18} \mathrm{O}$ in emission without clear absorption. ${ }^{(d)}$ From ${ }^{13} \mathrm{CO} .{ }^{(e)}$ From $\mathrm{H}_{2} \mathrm{O}$.

small volume $\left(\lesssim 20^{\prime \prime}\right)$ from the source, unlike the outflow gas at lower temperature and density traced by low- $J \mathrm{CO}$ lines.

We measured the size of the $987 \mathrm{GHz}$ emission by fitting a two-dimensional Gaussian plus a background offset to the images in Figs. 2 and A.1-A.17. Table 4 reports the resulting radii, which have been deconvolved assuming that the source and beam profiles add in quadrature. The measured sizes of the $\mathrm{H}_{2} \mathrm{O}$ emission are $\sim 2 \times$ smaller than the values measured in high- $J$ CO lines with Herschel (Karska et al. 2014; Kwon et al. 2017), and $2-3 \times$ smaller than the sizes of the submillimeter dust emission measured from the ground (Van der Tak et al. 2013). Evidently, the $\mathrm{H}_{2} \mathrm{O}$ emission traces warm dense gas close to the protostars.

Comparing the major and minor axis values in Table 4, we see that the $\mathrm{H}_{2} \mathrm{O}$ emission is close to spherical in most cases, with axis ratios between 1.1 and 1.4. We conclude that protostellar envelopes dominate the emission, without any evidence for flattening or elongation caused by rotation or bipolar outflows.

Table 4 compares the observed shape of the $\mathrm{H}_{2} \mathrm{O} 987 \mathrm{GHz}$ emission to the predictions from radiative transfer models, assuming a constant $\mathrm{H}_{2} \mathrm{O}$ abundance, following Herpin et al. (2016). These predictions are fits to multi-line $\mathrm{H}_{2} \mathrm{O}$ (and isotopic) spectra from HIFI, using the physical structure models from Van der Tak et al. (2013). The predicted size is seen to be $30-40 \%$ larger than the observed size for most sources, which we consider good agreement given the simplifying assumption of spherical symmetry in the models. Only for the sources W3 IRS5 and W43 MM1, the predicted size is 2-4 times smaller than the observed size. As with the axis ratios, this may be due to outflows contributing to the emission. Furthermore, the models assume a single central source, whereas interferometric images of our objects often show multiple cores at the center (e.g., Hunter et al. 2014; Brogan et al. 2016; Izquierdo et al. 2018).

The line intensities in the maps are typically $70-80 \%$ of the values reported from pointed observations at the same position.
This difference is as expected from the 4\% larger beam size due to the OTF observing mode and the spatial regridding, assuming a small emitting area. Only for IRAS 18151 and IRAS 18089 , the map intensities are substantially lower $(\approx 40 \%$ of the pointed observations) for unknown reasons. In such cases, the pointed observations are more reliable, since their calibration is more thorough, with multiple references and longer integrations. We conclude that mapping modes are useful to measure source sizes, but usually underestimate line intensities, sometimes substantially.

\section{Discussion}

\subsection{Origin of $\mathrm{H}_{2} \mathrm{O}$ and $\mathrm{H}_{2}^{18} \mathrm{O}$ line emission and absorption}

Figure 3 compares the observed $\mathrm{H}_{2}^{18} \mathrm{O}$ line profiles with those of the $\mathrm{H}_{2} \mathrm{O} 987$ and $752 \mathrm{GHz}$ lines. For the $987 \mathrm{GHz}$ line, we use the pointed observations rather than convolving the map data, because of the calibration issue with the maps (Sect. 3.2) and because the map data have higher noise levels. Remarkably, the $\mathrm{H}_{2}^{18} \mathrm{O}$ line profile (shown in black) is very similar to the difference between the two $\mathrm{H}_{2} \mathrm{O}$ lines (shown in gray). As found before for the case of W43-MM1 by another method (Jacq et al. 2016), this close similarity implies that the $\mathrm{H}_{2}^{18} \mathrm{O}$ absorption originates in warm gas $(T \gtrsim 100 \mathrm{~K})$. Given the upper level energies of the two $\mathrm{H}_{2} \mathrm{O}$ lines ( 101 and $137 \mathrm{~K}$ ), the bulk of the $\mathrm{H}_{2}^{18} \mathrm{O}$ absorption must arise in gas with temperatures between $\sim 100$ and $\sim 140 \mathrm{~K}$. These temperatures are just above the point where $\mathrm{H}_{2} \mathrm{O}$ ice sublimates from dust grains, which is expected to lead to a strong increase in the gas-phase $\mathrm{H}_{2} \mathrm{O}$ abundance (Boogert et al. 2015). The $\mathrm{H}_{2}^{18} \mathrm{O}$ absorption is unlikely to arise in the cold outer envelope, where the $\mathrm{H}_{2} \mathrm{O}$ abundance is too low to create detectable absorption in $\mathrm{H}_{2}^{18} \mathrm{O}$ (cf. Shipman et al. 2014). The success of the subtraction procedure shows that the outer envelope does not contribute to the $\mathrm{H}_{2}^{18} \mathrm{O}$ absorption.

For the sources W3 IRS5, NGC 7538, W33A, AFGL 2591, $\mathrm{G} 29.96$, G10.47, and W51N, the subtraction also reproduces $\mathrm{H}_{2}^{18} \mathrm{O}$ emission features. Since emission is sensitive to beam filling factors, this similarity is even stronger evidence that the $\mathrm{H}_{2}^{18} \mathrm{O}$ line originates between the layers where the $752 \mathrm{GHz}$ line is excited and where the $987 \mathrm{GHz}$ line is excited. In the models by Van der Tak et al. (2013), this zone occurs typically at radii of 1000-5000 au, depending on the luminosity of the source. This region is small enough that it is often difficult to observe (e.g., $2-10^{\prime \prime}$ diameter at a distance of $1 \mathrm{kpc}$ ).

In some cases, scaling the $752 \mathrm{GHz}$ profile before subtracting it from the $987 \mathrm{GHz}$ line profile improves the match of the difference to the $\mathrm{H}_{2}^{18} \mathrm{O}$ profile (Fig. 4), in particular for the line wings. The scaling factors that best match the observed profiles range from $\approx 1$ for sources with small deconvolved sizes (Table 4 ) to $\approx 1.8$ for the most extended sources. These values are just as expected from beam size differences between the 987 and $752 \mathrm{GHz}$ spectra, assuming equal excitation temperatures. There may be other pairs of lines whose differences enable us to probe specific layers of the protostellar cores.

Toward several of the more massive sources, the $\mathrm{H}_{2}^{18} \mathrm{O}$ line profiles show absorption in the line wings, especially on the blue-shifted side. Clearly, the $\mathrm{H}_{2}^{18} \mathrm{O}$ column density is sufficient to absorb even at velocities only seen in the wings. The $\mathrm{C}^{18} \mathrm{O}$ 9-8 spectra show no such high-velocity signals, which implies that the $\mathrm{H}_{2} \mathrm{O}$ abundance is enhanced in the highvelocity gas (Herpin et al. 2016). For example, the $\mathrm{H}_{2}^{18} \mathrm{O}$ spectrum toward NGC $6334 \mathrm{I}(\mathrm{N})$ shows absorption out to at least $15-20 \mathrm{~km} \mathrm{~s}^{-1}$ from line center, which has no counterpart in 
IRAS 05358 +3543
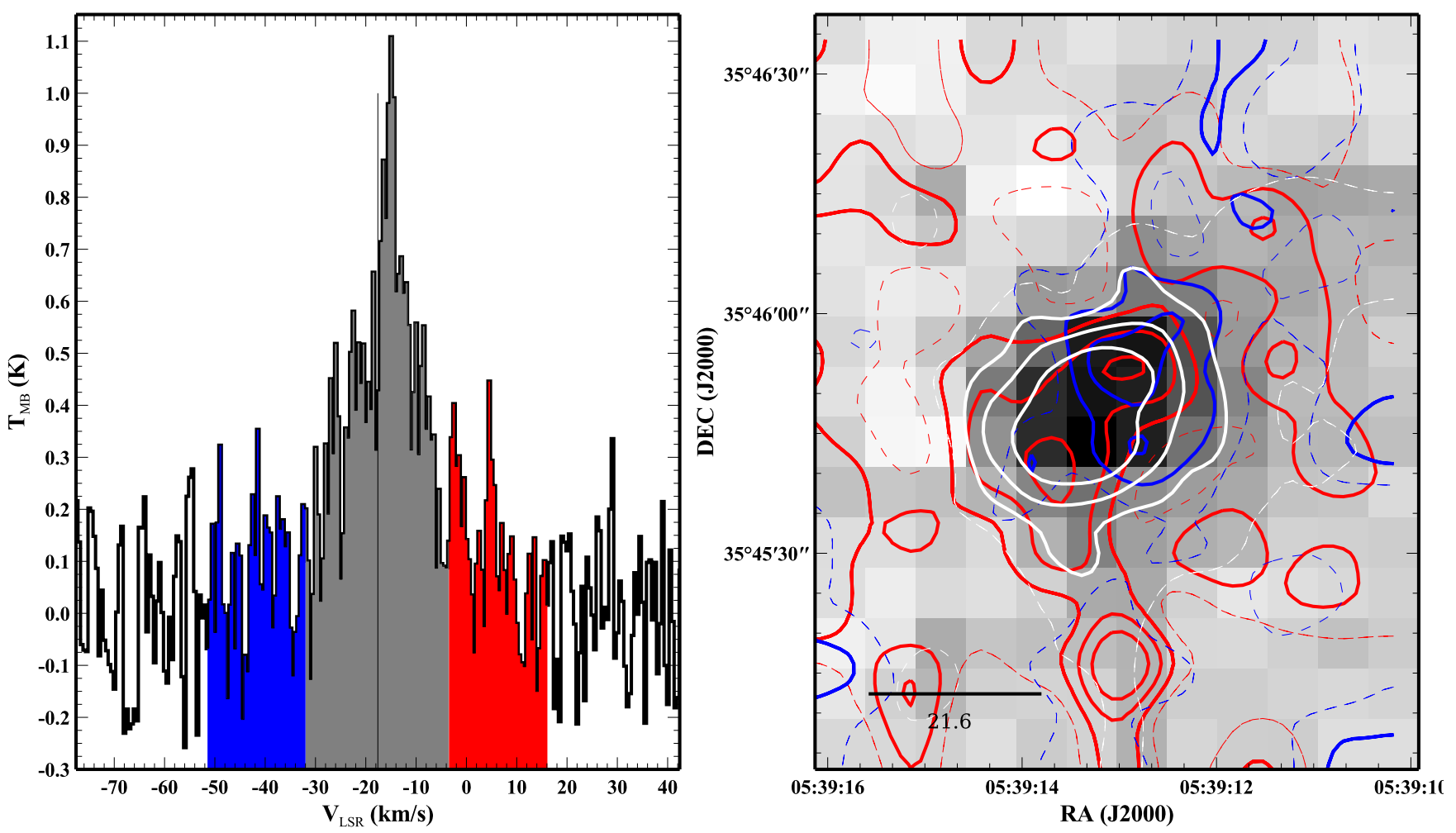

Fig. 2. Map of the velocity-integrated emission in the $\mathrm{H}_{2} \mathrm{O} 987 \mathrm{GHz}$ line for IRAS 05358. White contours and grayscale image denote velocityintegrated emission over the range indicated by the gray area in the spectrum in the left panel. Red and blue contours denote red-and blueshifted emission, indicated by the red and blue areas in the left panel. The red and blue maps were created by integrating the $987 \mathrm{GHz}$ data cube over a range of $1 F W H M$ below and above the $V_{\mathrm{LSR}}$ of the envelope, denoted by the vertical black line in the spectrum. The integration ranges are offset by $1 / 2 F W H M$ from the $V_{\text {LSR }}$ to avoid confusion with absorption features. The lowest contour (at the $1 \sigma$ level) is drawn dashed, the others (in multiples of $\sigma$ ) are drawn solid. The bar in the bottom left corner denotes the HIFI beam size.

$\mathrm{C}^{18} \mathrm{O}$. For this source, the integrated $\mathrm{H}_{2}^{18} \mathrm{O}$ absorption from the envelope (roughly between -6 and $+1 \mathrm{~km} \mathrm{~s}^{-1}$, which has a counterpart in $\mathrm{C}^{18} \mathrm{O}$ emission) is approximately equal to that in the high-velocity blue wing. In contrast, the $\mathrm{C}^{18} \mathrm{O} 9-8$ line indicates $\gtrsim 10 \times$ less mass at high velocities, implying an $\mathrm{H}_{2} \mathrm{O}$ abundance enhancement by more than an order of magnitude.

Similar conclusions hold for the other sources, except for G5.89 and G34.26 for which weak wings are seen on the $\mathrm{C}^{18} \mathrm{O}$ 9-8 profiles. The lack of high-velocity $\mathrm{C}^{18} \mathrm{O} 9-8$ emission for most sources is not an excitation effect, as low- $J \mathrm{C}^{18} \mathrm{O}$ lines do not show wings either (Hatchell et al. 1998; Watson et al. 2003; Gibb et al. 2004; Thomas \& Fuller 2007). We conclude that $\mathrm{H}_{2} \mathrm{O}$ abundances in high-mass protostellar outflows are $\gtrsim 10 \times$ higher than in the envelopes.

The $\mathrm{H}_{2} \mathrm{O}$ abundance in these sources thus appears to have at least 3 levels: low in the outer envelope, high in the inner envelope, and very high in the outflow. This is in line with the work of Van der Tak et al. (2010), who used HIFI maps of the DR21 region in ${ }^{13} \mathrm{CO} 10-9$ and $\mathrm{H}_{2} \mathrm{O} 1_{11}-0_{00}$ to derive $\mathrm{H}_{2} \mathrm{O}$ abundances of $\sim 10^{-10}$ for the cool outer envelope, $\sim 10^{-8}$ for the warm inner envelope, and $\sim 10^{-6}$ for the shocked outflowing gas.

\subsection{Infall rates and trends}

The rightmost column of Table 3 gives estimates of the infall rates onto our sources. These were calculated using

$\dot{M}_{\mathrm{acc}}=4 \pi R^{2} m\left(\mathrm{H}_{2}\right) n\left(\mathrm{H}_{2}\right)\left|V_{\mathrm{inf}}\right|$, where $m\left(\mathrm{H}_{2}\right)$ is the mass of the $\mathrm{H}_{2}$ molecule, and the absolute value of the infall speed $V_{\text {inf }}$ is taken from Table 3 . Infall motion appears negative as gas is moving toward the center of the reference frame of our models. Given the similarity of the $\mathrm{H}_{2}^{18} \mathrm{O}$ absorption profile with the difference of the $\mathrm{H}_{2} \mathrm{O} 987$ and $752 \mathrm{GHz}$ profiles (Sect. 4.1), we adopt the radius of the $120 \mathrm{~K}$ point in the envelope models from Van der Tak et al. (2013) for $R$, and the density at that radius for $n\left(\mathrm{H}_{2}\right)$. These radii vary between 800 and $9000 \mathrm{au}$, and the densities from $7 \times 10^{5}$ to $5 \times 10^{7} \mathrm{~cm}^{-3}$. Our observed (deconvolved) sizes agree well (within a factor of 2) with the upper end of this range, except for W43 MM1, G10.47, and W51N, where the observed values are larger.

The resulting infall rates (Table 3 , right column) are seen to range from $\sim 7 \times 10^{-5}$ to $\sim 2 \times 10^{-2} M_{\odot} \mathrm{yr}^{-}$. These values are in reasonable agreement with other observations (e.g., König et al. 2017) and with theoretical models (Tan et al. 2014; Motte et al. 2018). They should be considered order of magnitude estimates, because of our simplified treatment assuming spherical symmetry. The observational uncertainty through the measured line velocities is only a $\sim 30 \%$ effect. The derived infall rates depend only weakly on the adopted radius and density: the envelopes of our sources have density profiles that drop off approximately as $R^{-2}$, so that the effects of $R$ and $n$ on $\dot{M}$ tend to cancel each other.

For our subsamples of mid-infrared quiet and -bright HMPOs, Herpin et al. (2016) and Choi (2015) have made detailed models of the $\mathrm{H}_{2} \mathrm{O}$ distribution in the protostellar envelope, including simple step functions for the $\mathrm{H}_{2} \mathrm{O}$ abundance in the inner and outer envelope. In order to fit the line profiles of $\mathrm{H}_{2} \mathrm{O}$, 
Table 4. Observed and deconvolved source sizes (arcsec).

\begin{tabular}{|c|c|c|c|c|c|c|}
\hline Source ${ }^{(a)}$ & Major axis & Minor axis & Position angle (degrees) & Deconvolved $^{(b)}$ & Model $^{(c)}$ & $850-870 \mu \mathrm{m}^{(d)}$ \\
\hline IRAS 05358 & $12.0(0.5)$ & $9.3(0.4)$ & $-46(6)$ & 4.4 & 15.0 & 30.0 \\
\hline IRAS 16272 & $15.9(0.7)$ & $11.8(0.6)$ & $-34(5)$ & 8.8 & 18.0 & 50.0 \\
\hline NGC $6334 \mathrm{I}(\mathrm{N})$ & $17.2(0.5)$ & $12.5(0.4)$ & $38(3)$ & 11.3 & 21.6 & 42.0 \\
\hline W43 MM1 & $15.8(1.6)$ & $12.8(1.2)$ & $-89(15)$ & 15.5 & 11.8 & 27.0 \\
\hline $\mathrm{DR} 21(\mathrm{OH})$ & $12.4(0.4)$ & $11.1(0.4)$ & $-8(11)$ & 5.4 & 28.8 & 33.0 \\
\hline W3 IRS5 & $14.1(0.3)$ & $12.0(0.3)$ & $-9(5)$ & 7.8 & 40.0 & 57.0 \\
\hline IRAS 18089 & $11.0(0.9)$ & $9.5(0.8)$ & $-37(21)$ & $-0-$ & $\ldots$ & 17.0 \\
\hline W33A & $11.6(0.7)$ & $10.0(0.6)$ & $-44(16)$ & 5.1 & $\ldots$ & 30.0 \\
\hline AFGL 2591 & $11.3(0.6)$ & $9.7(0.5)$ & $76(12)$ & 3.8 & 25.2 & 25.2 \\
\hline G327-0.6 & $14.3(0.5)$ & $10.2(0.4)$ & $67(4)$ & 5.5 & $\ldots$ & 24.0 \\
\hline NGC 6334I & $16.1(0.5)$ & $15.0(0.4)$ & $88(13)$ & 12.6 & $\ldots$ & 40.0 \\
\hline G29.96 & $10.7(0.3)$ & $9.6(0.3)$ & $-13(11)$ & 3.0 & $\ldots$ & 16.0 \\
\hline G31.41 & $9.9(0.9)$ & $7.5(0.7)$ & $-5(13)$ & $-0-$ & $\ldots$ & 15.0 \\
\hline G5.89 & $10.9(0.2)$ & $10.0(0.2)$ & $-29(7)$ & 3.9 & $\ldots$ & 28.0 \\
\hline G10.47 & $11.9(0.7)$ & $10.2(0.6)$ & $-47(14)$ & 5.5 & $\ldots$ & 10.0 \\
\hline G34.26 & $22.4(1.0)$ & $16.9(0.8)$ & $-17(4)$ & 16.9 & $\ldots$ & 25.0 \\
\hline W51N-e1 & $14.5(0.7)$ & $10.9(0.5)$ & $-63(6)$ & 8.2 & $\ldots$ & 27.0 \\
\hline
\end{tabular}

Notes. ${ }^{(a)}$ The source fitting failed for IRAS 18151 and NGC 7538. ${ }^{(b)}$ Equivalent circular axis; a value of zero means that the source is unresolved. ${ }^{(c)}$ From Chavarría et al. (2010) for W3 IRS5, Herpin (priv. comm.) for AFGL 2591; other sources from Herpin et al. (2016). ${ }^{(d)} 3 \sigma$ radii from Van der Tak et al. (2013).
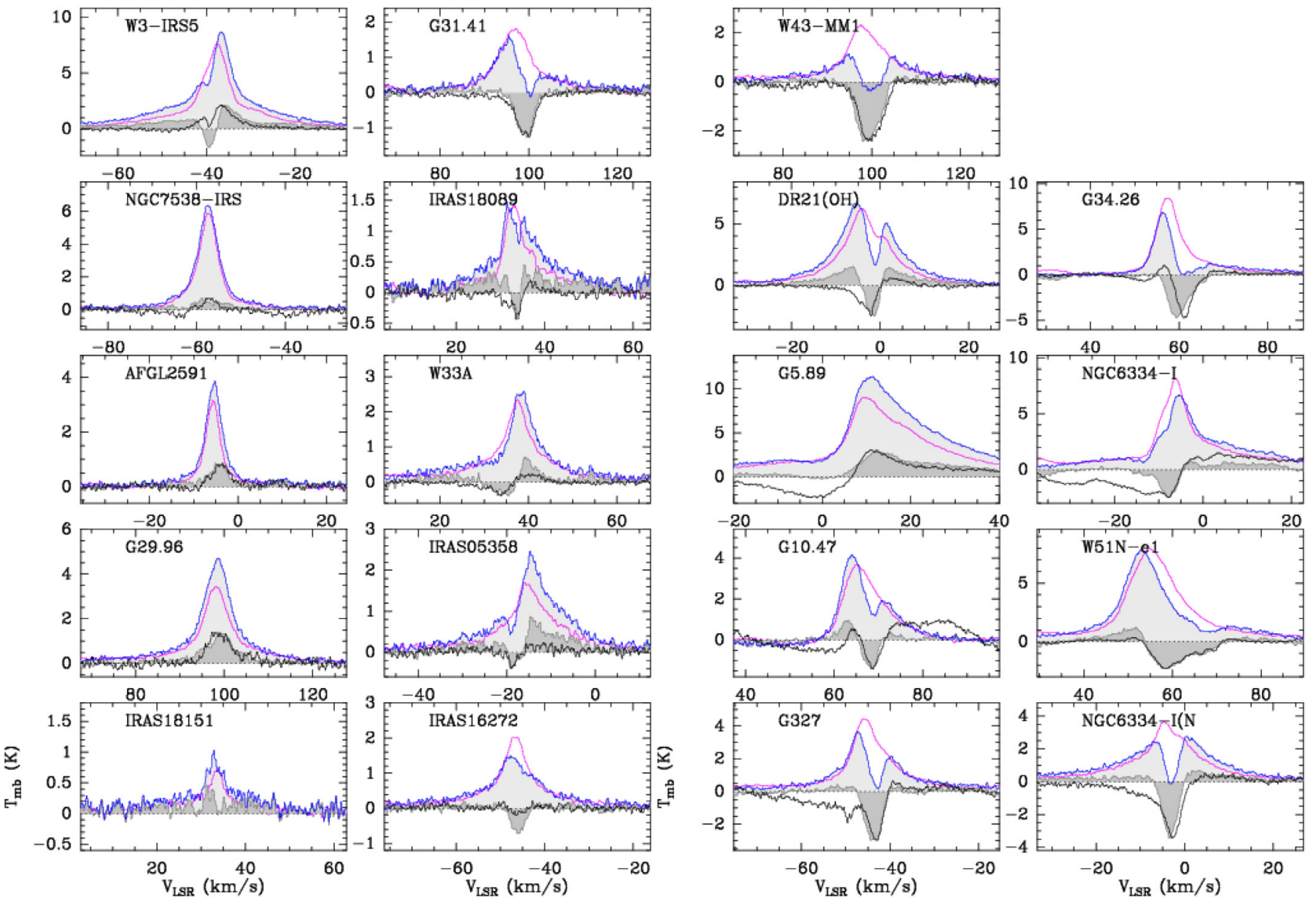

Fig. 3. Spectra of the $\mathrm{H}_{2} \mathrm{O} 987$ and $752 \mathrm{GHz}$ lines (blue and purple histograms), and their difference (shaded gray histogram), compared with the $\mathrm{H}_{2}^{18} \mathrm{O}$ line profile (black) histogram. 


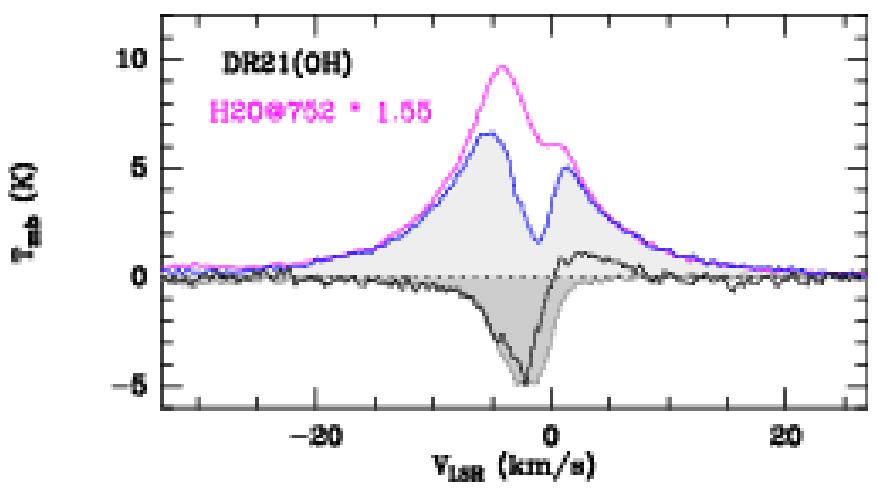

Fig. 4. As previous figure, for $\mathrm{DR} 21(\mathrm{OH})$, with the $752 \mathrm{GHz}$ profile scaled to optimize the match to the $\mathrm{H}_{2}^{18} \mathrm{O}$ line wings.

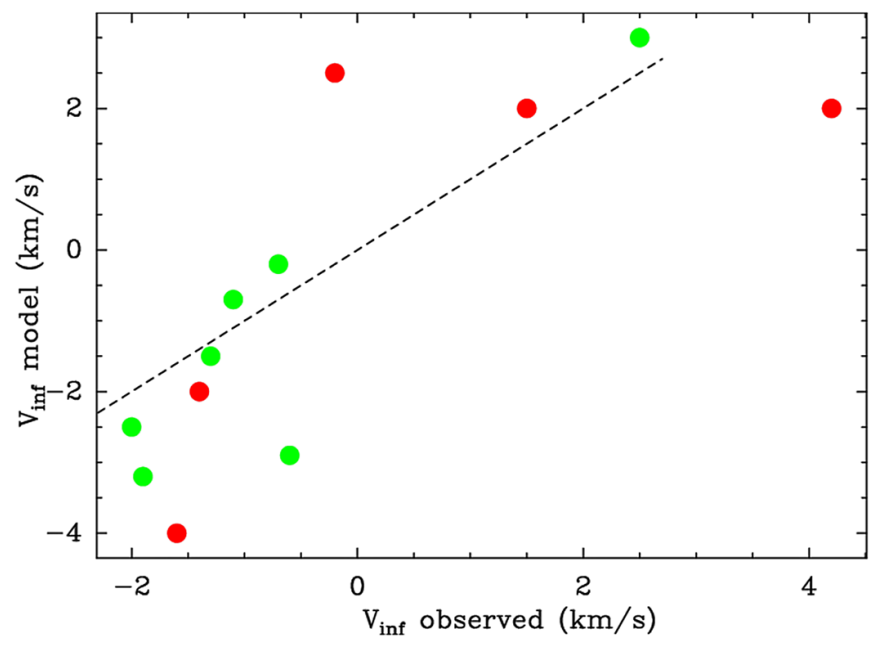

Fig. 5. Infall velocities estimated from peak shift between $\mathrm{H}_{2}^{18} \mathrm{O}$ and $\mathrm{C}^{18} \mathrm{O}$ lines vs. values from detailed fits to $\mathrm{H}_{2} \mathrm{O}$ line profiles (using RATRAN) by Herpin et al. (2016) (green) and Choi (2015) (red). The dashed line denotes 1:1 correspondence.

$\mathrm{H}_{2}^{18} \mathrm{O}$, and $\mathrm{H}_{2}^{17} \mathrm{O}$ in the pointed HIFI spectra, Herpin and Choi had to include radial motions in their models. Figure 5 compares their derived infall velocities to the values found in this work; the dashed line indicates 1:1 correspondence. The two types of estimates of the radial velocity are seen to agree qualitatively, both in the sign of the velocity (infall or outflow) and in its magnitude. The simple estimates of the inflow/expansion velocity are on average $\approx 2 \times$ lower than those from the detailed models, although for four sources, they are actually larger. We consider this agreement as reasonable, given the differences between the two approaches.

Since our source sample covers a range of luminosities, envelope masses, and evolutionary stages, we investigated if our derived source sizes, infall velocities, and infall rates show any trends with $L_{\mathrm{bol}}, M_{\mathrm{env}}$, and age. To estimate the relative ages of our sources, we used the ratio of $L_{\mathrm{bol}} / M_{\mathrm{env}}$, which is straightforward to compute and appears to be a robust age tracer (Molinari et al. 2016). In addition, we used the presence of hot molecular cores and/or ultracompact HII regions as a sign of a relatively evolved stage. Third, we looked for trends with the virial mass and the ratio $M_{\mathrm{vir}} / M_{\mathrm{env}}$, proposed as a stability parameter by König et al. (2017). Virial masses are calculated following Giannetti et al. (2014), using line widths from Table A.2 of Van der Tak et al. (2013).
The only significant trend that we find is between the linear sizes of our sources with their virial masses. Since virial mass depends on size, this trend probably just means that the line width is similar for all sources. In addition, the infall rates seem to increase with virial mass and with the evolutionary indicator $L_{\mathrm{bol}} / M_{\mathrm{env}}$, but the statistical significance of these trends is small. Even the relation with $M_{\mathrm{vir}}$ has a Pearson correlation coefficient of only $r=0.58$. For a sample size of $N=11$, this $r$-value corresponds to a probability of false correlation of $p=6 \%$, i.e. $\mathrm{a} \approx 2 \sigma$ significance. We conclude that the accretion rates may increase with circumstellar mass and with evolutionary stage, but that larger source samples are required to test these claims.

\section{Conclusions}

Based on our measured velocity shifts between $\mathrm{H}_{2}^{18} \mathrm{O}$ absorption and $\mathrm{C}^{18} \mathrm{O}$ emission, infall motions appear to be common in the embedded phase of high-mass star formation, at typical accretion rates of $\sim 1 \times 10^{-4} M_{\odot} \mathrm{yr}^{-1}$. We find a tentative trend that the highest accretion rates occur for the most massive sources, which is globally consistent with current models of high-mass star formation (Tan et al. 2014; Motte et al. 2018). Our data do not allow us to distinguish between such models, however.

In addition, the accretion rates may increase with age, unlike in the low-mass case, for which accretion rates drop from the Class 0 to the Class III stage, and are highly episodic (Dunham et al. 2014). Signs of episodic accretion, which is well established in the low-mass case, have recently been reported for a high-mass star, in the form of mid-infrared variability suggesting accretion "bursts" (Caratti o Garatti et al. 2017).

Our data do not allow us to discern trends within specific types of sources, nor with protostellar luminosity. A study of $\mathrm{H}_{2} \mathrm{O}$ line profiles toward a large $(N \sim 100)$ sample is needed to distinguish such trends and to search for episodic behavior. Data from the Herschel open time programs by Bontemps and Wyrowski may be suitable for this purpose. In the future, such studies will be possible with ESA's SPace Infrared telescope for Cosmology and Astrophysics (SPICA) ${ }^{3}$ (Roelfsema et al. 2018; Van der Tak et al. 2018) around 2030, and NASA's Origins Space Telescope (OST) ${ }^{4}$ (Battersby et al. 2018) around 2040.

Acknowledgements. This paper is dedicated to the memory of Malcolm Walmsley, who passed away on 1 May 2017 at the age of 75. We remember Malcolm as a great source of inspiration, and we will miss his sharp insight and kind manner. The authors thank the WISH team led by Ewine van Dishoeck for inspiring discussions, and the anonymous referee for useful comments on the manuscript. This research has used the following databases: ADS, CDMS, JPL, and LAMDA. HIFI was designed and built by a consortium of institutes and university departments from across Europe, Canada and the US under the leadership of SRON Netherlands Institute for Space Research, Groningen, The Netherlands with major contributions from Germany, France and the USA. Consortium members are: Canada: CSA, U.Waterloo; France: CESR, LAB, LERMA, IRAM; Germany: KOSMA, MPIfR, MPS; Ireland, NUI Maynooth; Italy: ASI, IFSI-INAF, Arcetri-INAF; Netherlands: SRON, TUD; Poland: CAMK, CBK; Spain: Observatorio Astronómico Nacional (IGN), Centro de Astrobiología (CSIC-INTA); Sweden: Chalmers University of Technology - MC2, RSS \& GARD, Onsala Space Observatory, Swedish National Space Board, Stockholm University - Stockholm Observatory; Switzerland: ETH Zürich, FHNW; USA: Caltech, JPL, NHSC.

\section{References}

Battersby, C., Armus, L., Bergin, E., et al. 2018, Nat. Astron., 2, 596 Beltrán, M. T., \& de Wit, W. J. 2016, A\&ARv, 24, 6 Beltrán, M. T., Cesaroni, R., Codella, C., et al. 2006, Nature, 443, 427

$\begin{array}{ll}3 & \text { http://www.spica-mission.org/ } \\ 4 & \text { https://asd.gsfc.nasa.gov/firs/ }\end{array}$ 
Beltrán, M. T., Cesaroni, R., Rivilla, V. M., et al. 2018, A\&A, 615, A141 Beuther, H., Schilke, P., Sridharan, T. K., et al. 2002, A\&A, 383, 892

Boogert, A. C. A., Gerakines, P. A., \& Whittet, D. C. B. 2015, ARA\&A, 53, 541

Brogan, C. L., Hunter, T. R., Cyganowski, C. J., et al. 2016, ApJ, 832, 187

Caratti o Garatti, A., Stecklum, B., Garcia Lopez, R., et al. 2017, Nat. Phys., 13, 276

Cesaroni, R., Sánchez-Monge, Á., Beltrán, M. T., et al. 2017, A\&A, 602, A59

Chavarría, L., Herpin, F., Jacq, T., et al. 2010, A\&A, 521, L37

Choi, Y. 2015, Ph.D. Thesis, University of Groningen

Churchwell, E., Walmsley, C. M., \& Cesaroni, R. 1990, A\&AS, 83, 119

Cunningham, N., Lumsden, S. L., Moore, T. J. T., Maud, L. T., \& Mendigutía, I. 2018, MNRAS, 477, 2455

De Graauw, T., Helmich, F. P., Phillips, T. G., et al. 2010, A\&A, 518, L6

Dunham, M. M., Stutz, A. M., Allen, L. E., et al. 2014, Protostars and Planets VI (Tucson: University of Arizona Press), 195

Endres, C. P., Schlemmer, S., Schilke, P., Stutzki, J., \& Müller, H. S. P. 2016, J. Mol. Spectr., 327, 95

Fuller, G. A., Williams, S. J., \& Sridharan, T. K. 2005, A\&A, 442, 949

Giannetti, A., Wyrowski, F., Brand, J., et al. 2014, A\&A, 570, A65

Gibb, A. G., Wyrowski, F., \& Mundy, L. G. 2004, ApJ, 616, 301

Hajigholi, M., Persson, C. M., Wirström, E. S., et al. 2016, A\&A, 585, A158

Hatchell, J., Thompson, M. A., Millar, T. J., \& MacDonald, G. H. 1998, A\&AS, 133, 29

He, Y.-X., Zhou, J.-J., Esimbek, J., et al. 2015, MNRAS, 450, 1926

Herpin, F., Chavarría, L., Jacq, T., et al. 2016, A\&A, 587, A139

Hunter, T. R., Brogan, C. L., Cyganowski, C. J., \& Young, K. H. 2014, ApJ, 788 187

Immer, K., Reid, M. J., Menten, K. M., Brunthaler, A., \& Dame, T. M. 2013, A\&A, 553, A117

Izquierdo, A. F., Galván-Madrid, R., Maud, L. T., et al. 2018, MNRAS, 478, 2505

Jacq, T., Braine, J., Herpin, F., van der Tak, F., \& Wyrowski, F. 2016, A\&A, 595, A66

Johnston, K. G., Robitaille, T. P., Beuther, H., et al. 2015, ApJ, 813, L19

Karska, A., Herpin, F., Bruderer, S., et al. 2014, A\&A, 562, A45

Klaassen, P. D., \& Wilson, C. D. 2007, ApJ, 663, 1092

König, C., Urquhart, J. S., Csengeri, T., et al. 2017, A\&A, 599, A139

Kurayama, T., Nakagawa, A., Sawada-Satoh, S., et al. 2011, PASJ, 63, 513

Kwon, W., van der Tak, F., Karska, A., et al. 2017, A\&A, submitted

Molinari, S., Merello, M., Elia, D., et al. 2016, ApJ, 826, L8

Motte, F., Schilke, P., \& Lis, D. C. 2003, ApJ, 582, 277

Motte, F., Bontemps, S., \& Louvet, F. 2018, ARA\&A, 56, 41
Mottram, J. C., van Dishoeck, E. F., Schmalzl, M., et al. 2013, A\&A, 558, A126 Nguyen Luong, Q., Motte, F., Schuller, F., et al. 2011, A\&A, 529, A41

Ott, S. 2010, in Astronomical Data Analysis Software and Systems XIX, eds. Y. Mizumoto, K.-I. Morita, \& M. Ohishi, ASP Conf. Ser., 434, 139 Peretto, N., André, P., \& Belloche, A. 2006, A\&A, 445, 979

Peters, T., Banerjee, R., Klessen, R. S., \& Mac Low, M.-M. 2011, ApJ, 729, 72 Pilbratt, G. L., Riedinger, J. R., Passvogel, T., et al. 2010, A\&A, 518, L1

Pillai, T., Kauffmann, J., Wyrowski, F., et al. 2011, A\&A, 530, A118

Reid, M. J., Menten, K. M., Brunthaler, A., et al. 2014, ApJ, 783, 130

Roelfsema, P. R., Helmich, F. P., Teyssier, D., et al. 2012, A\&A, 537, A17

Roelfsema, P. R., Shibai, H., Armus, L., et al. 2018, PASA, 35, e030

Rygl, K. L. J., Brunthaler, A., Sanna, A., et al. 2012, A\&A, 539, A79

Sánchez-Monge, Á., Cesaroni, R., Beltrán, M. T., et al. 2013, A\&A, 552, L10

San José-García, I., Mottram, J. C., Kristensen, L. E., et al. 2013, A\&A, 553, A125

San José-García, I., Mottram, J. C., van Dishoeck, E. F., et al. 2016, A\&A, 585 A103

Sanna, A., Reid, M. J., Menten, K. M., et al. 2014, ApJ, 781, 108

Sato, M., Reid, M. J., Brunthaler, A., \& Menten, K. M. 2010, ApJ, 720, 1055

Schöier, F. L., van der Tak, F. F. S., van Dishoeck, E. F., \& Black, J. H. 2005, A\&A, 432, 369

Shipman, R. F., van der Tak, F. F. S., Wyrowski, F., Herpin, F., \& Frieswijk, W. 2014, A\&A, 570, A51

Shipman, R. F., Beaulieu, S. F., Teyssier, D., et al. 2017, A\&A, 608, A49

Sollins, P. K., Zhang, Q., Keto, E., \& Ho, P. T. P. 2005, ApJ, 624, L49

Tan, J. C., Beltrán, M. T., Caselli, P., et al. 2014, Protostars and Planets VI (Tucson, AZ: University of Arizona Press), 149

Thomas, H. S., \& Fuller, G. A. 2007, ApJ, 659, L165

Van der Tak, F. F. S., Marseille, M. G., Herpin, F., et al. 2010, A\&A, 518, L107

Van der Tak, F. F. S., Chavarría, L., Herpin, F., et al. 2013, A\&A, 554, A83

Van der Tak, F. F. S., Madden, S. C., Roelfsema, P., et al. 2018, PASA, 35, e002

Van Dishoeck, E. F., Kristensen, L. E., Benz, A. O., et al. 2011, PASP, 123, 138

Watson, C., Araya, E., Sewilo, M., et al. 2003, ApJ, 587, 714

Wienen, M., Wyrowski, F., Menten, K. M., et al. 2015, A\&A, 579, A91

Wu, Y. W., Sato, M., Reid, M. J., et al. 2014, A\&A, 566, A17

Wyrowski, F., Güsten, R., Menten, K. M., Wiesemeyer, H., \& Klein, B. 2012, A\&A, 542, L15

Wyrowski, F., Güsten, R., Menten, K. M., et al. 2016, A\&A, 585, A149

Xu, J.-L., Li, D., Zhang, C.-P., et al. 2016, ApJ, 819, 117

Yang, B., Stancil, P. C., Balakrishnan, N., \& Forrey, R. C. 2010, ApJ, 718, 1062

Zhang, B., Moscadelli, L., Sato, M., et al. 2014, ApJ, 781, 89 


\section{Appendix A: Maps of all sources}

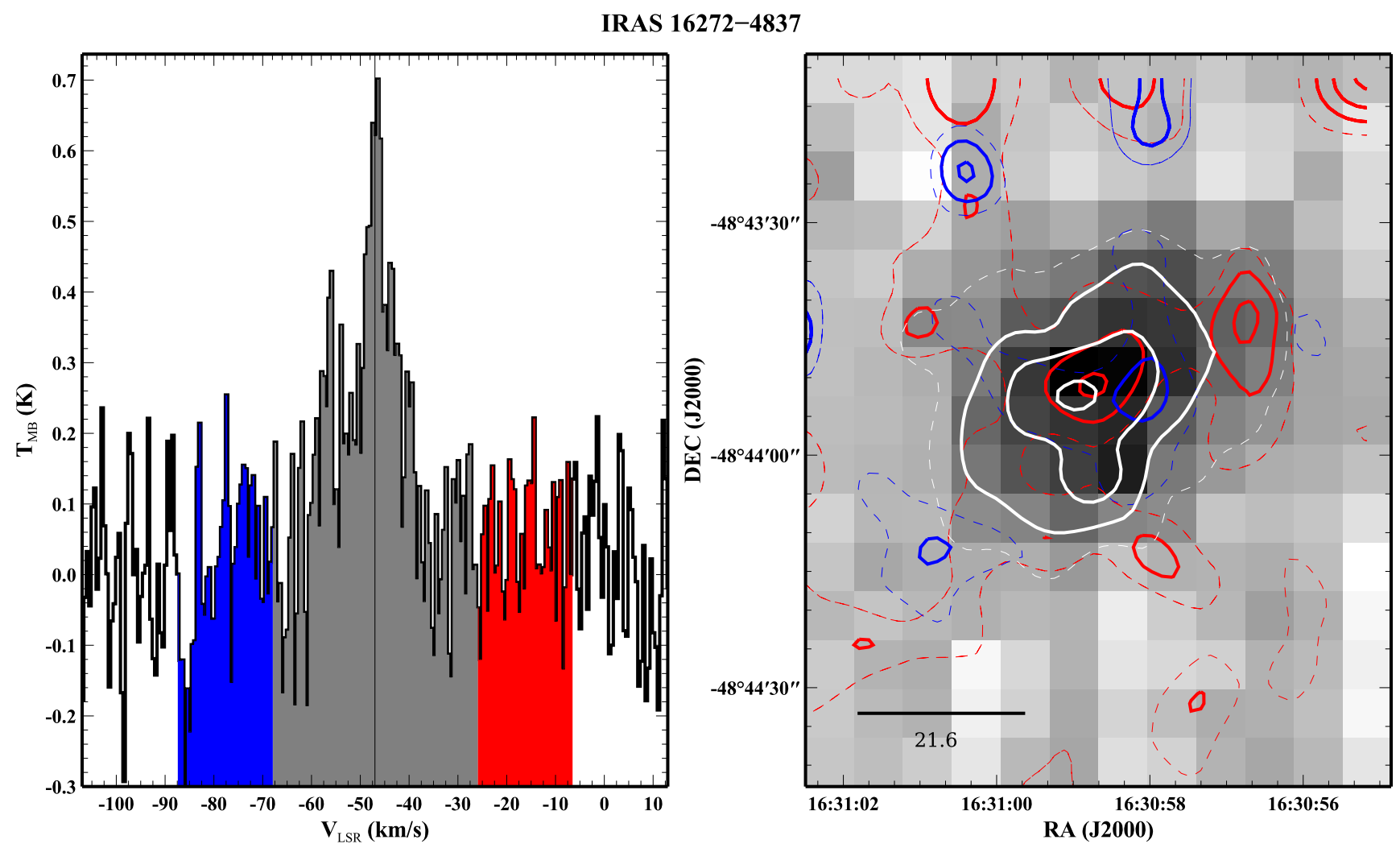

Fig. A.1. As Fig. 2, for IRAS 16272.
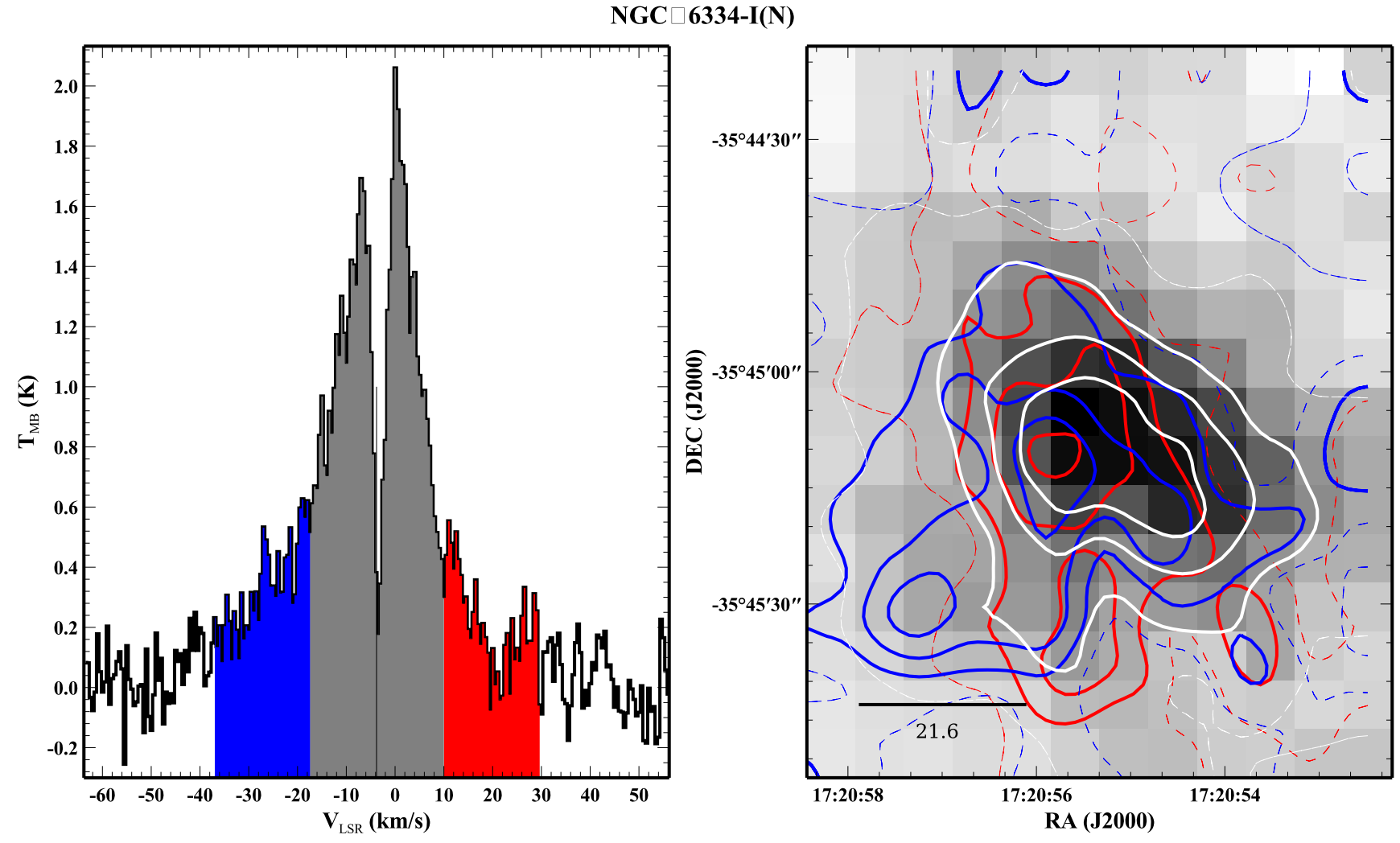

Fig. A.2. As previous figure, for NGC 6334I(N). 
W43-MM1
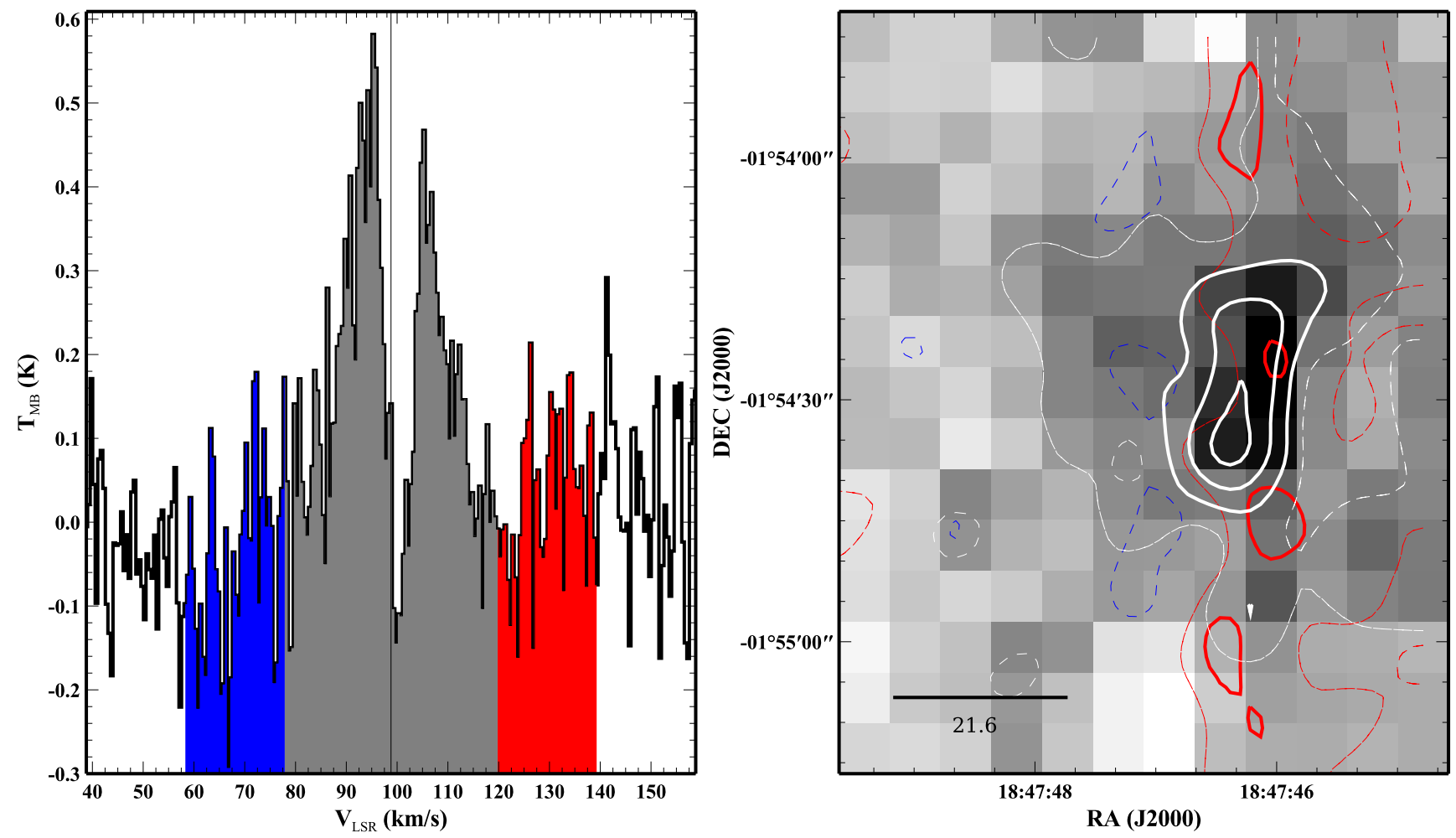

Fig. A.3. As previous figure, for W43-MM1.

DR21(OH)
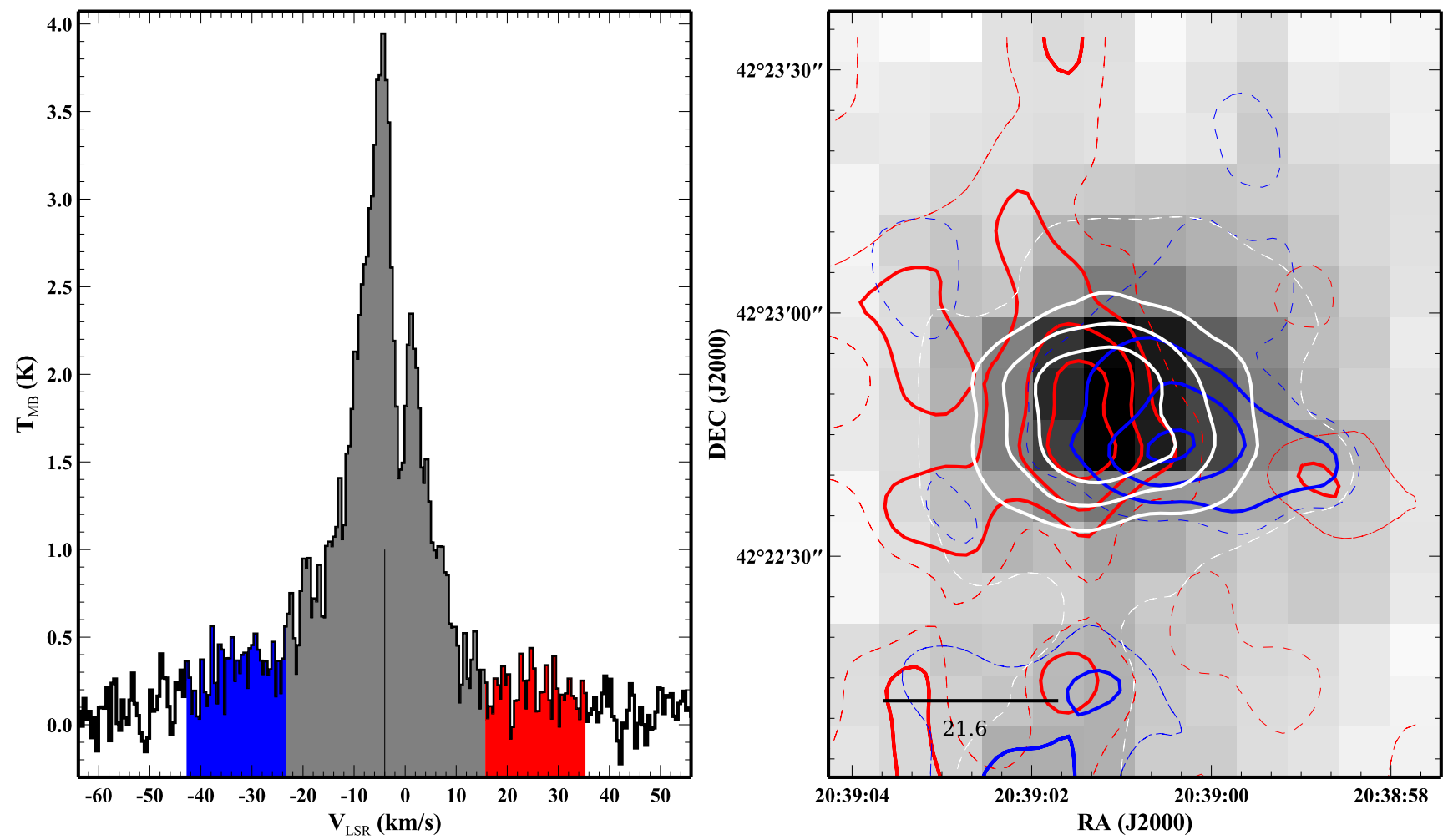

Fig. A.4. As previous figure, for DR21(OH). 
F. F. S. van der Tak et al.: Infall onto high-mass protostars

W3-IRS5
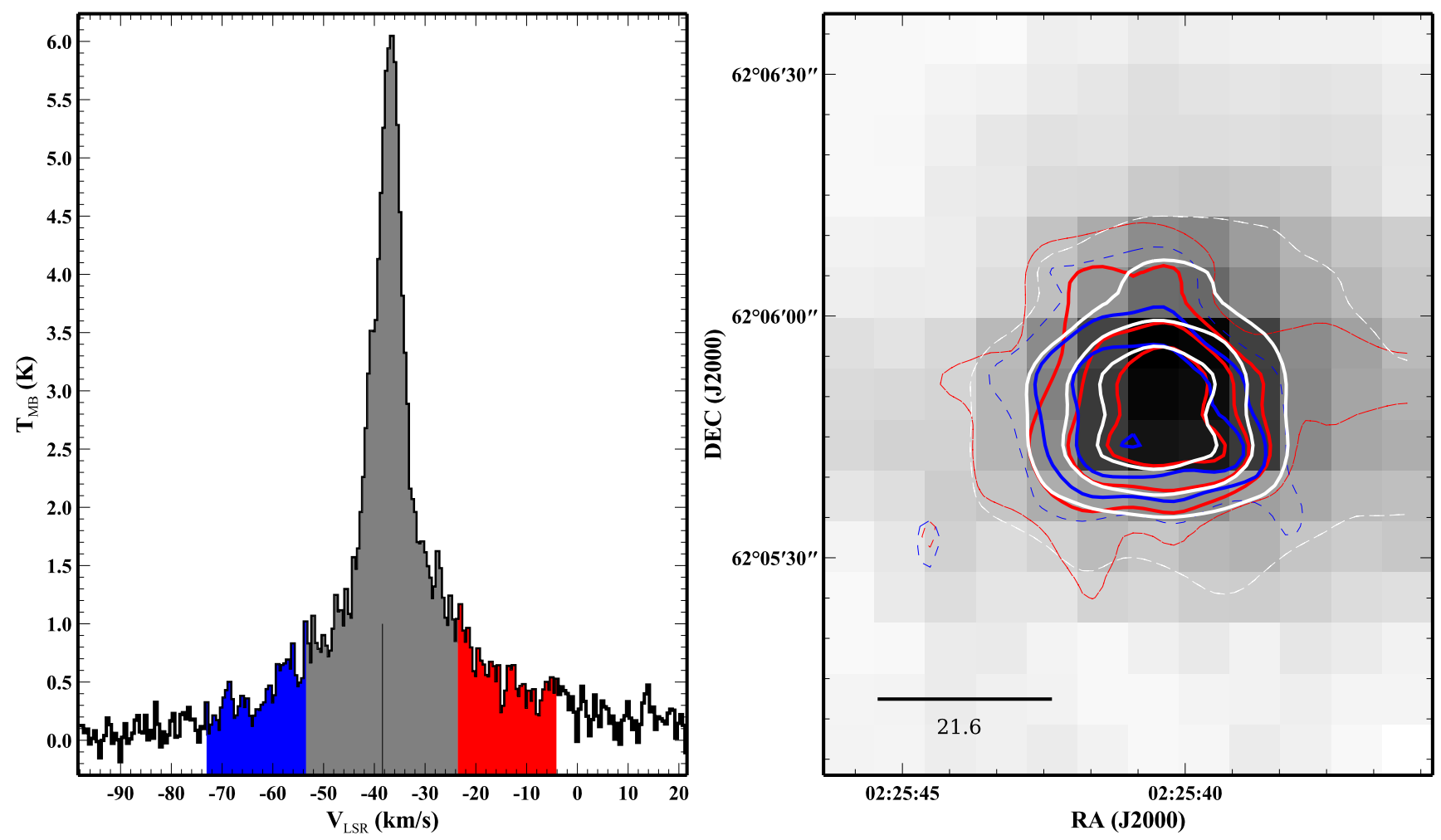

Fig. A.5. As previous figure, for W3 IRS5.

IRAS $\square$ 18089-1732
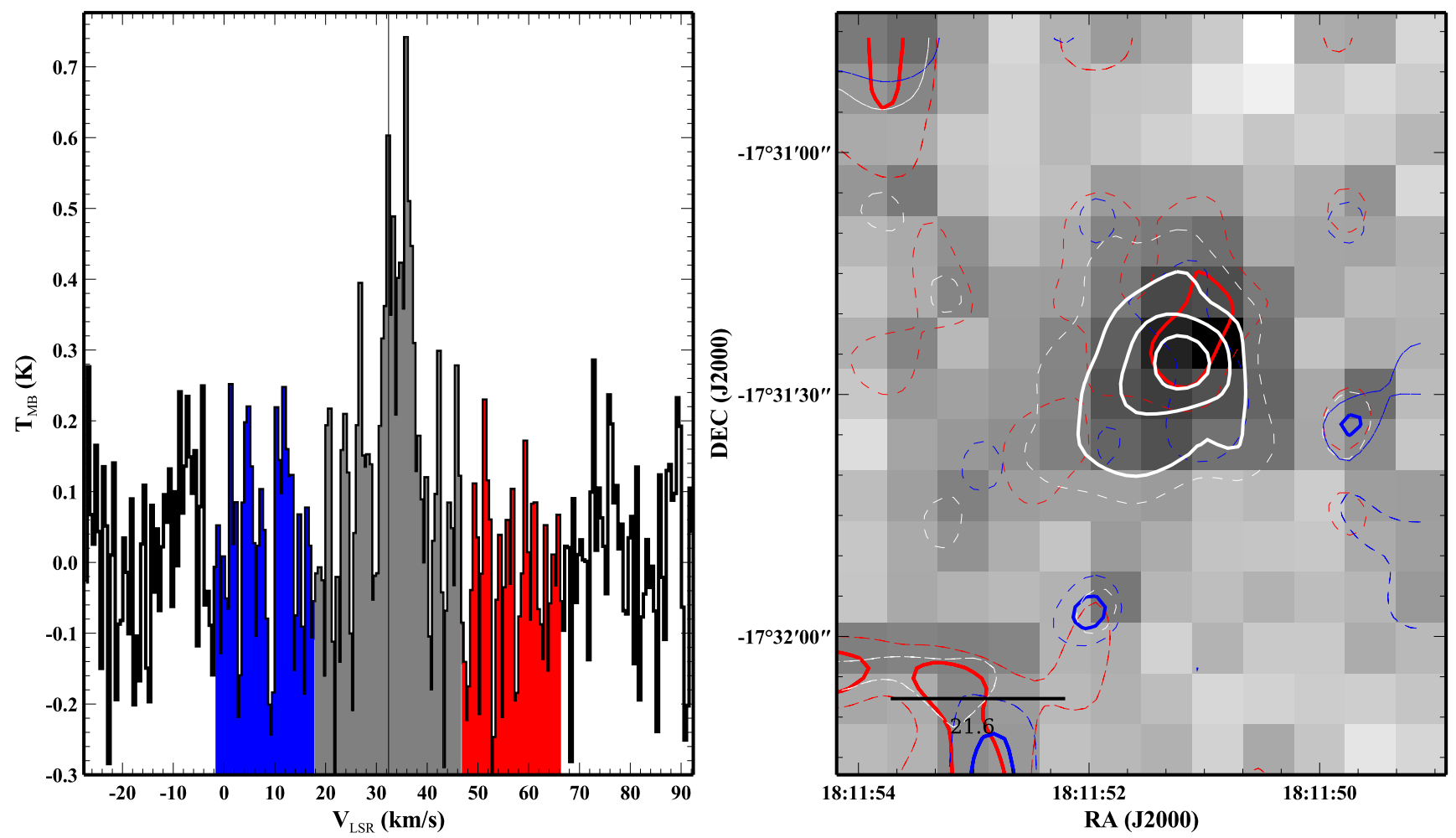

Fig. A.6. As previous figure, for IRAS 18089. 
W33A
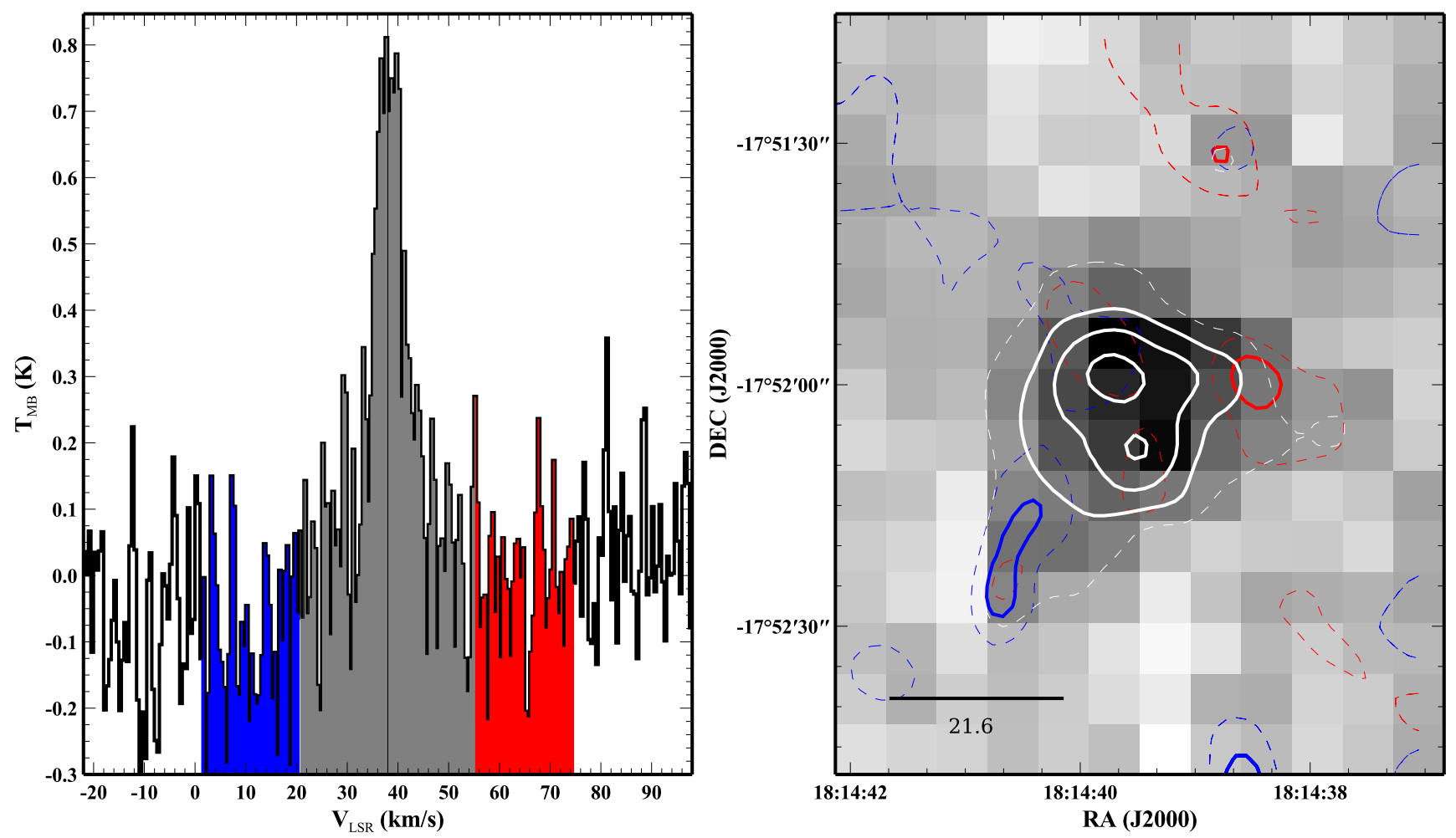

Fig. A.7. As previous figure, for W33A.
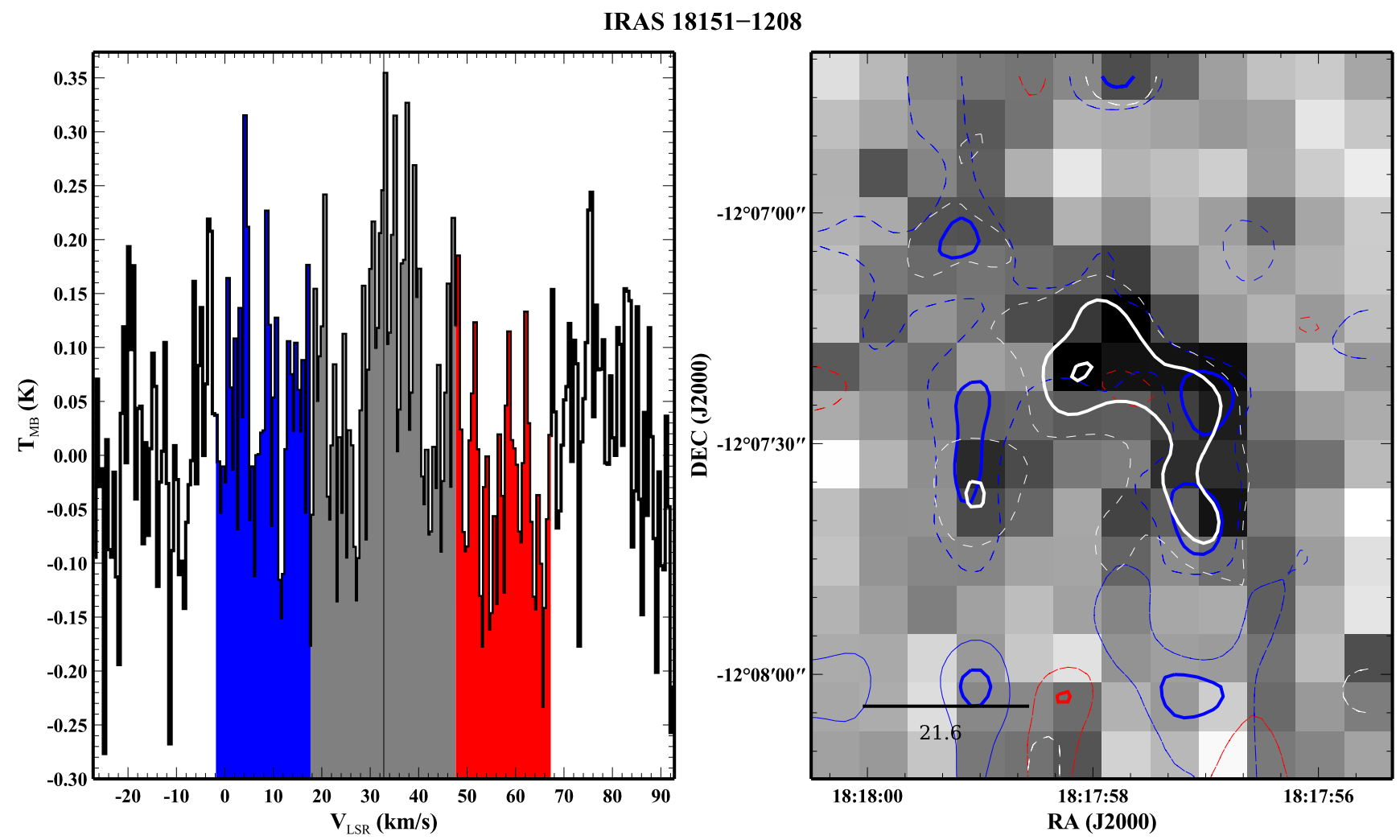

Fig. A.8. As previous figure, for IRAS 18151. 
F. F. S. van der Tak et al.: Infall onto high-mass protostars

AFGL2591
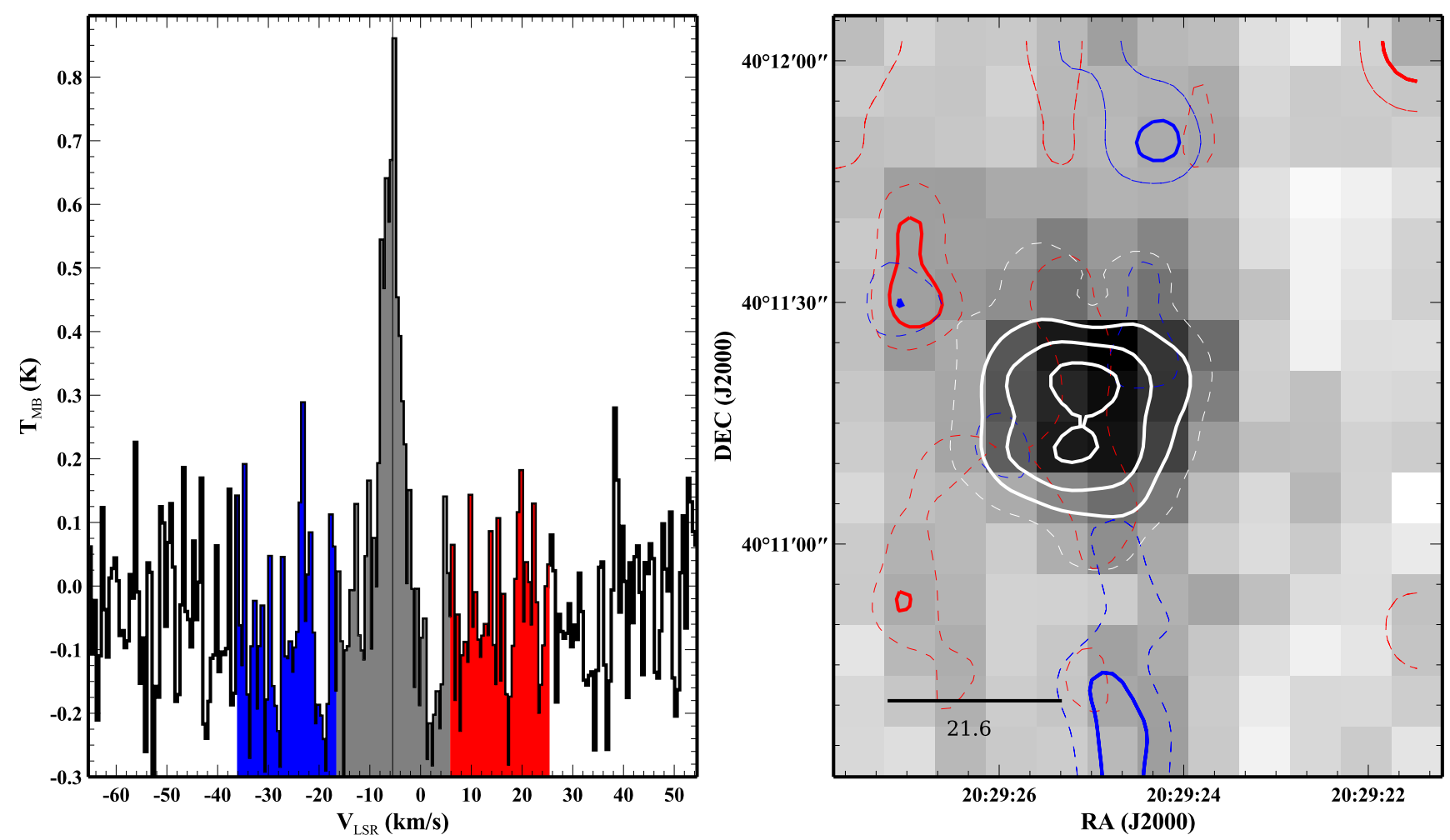

Fig. A.9. As previous figure, for AFGL 2591.

G327-0.6
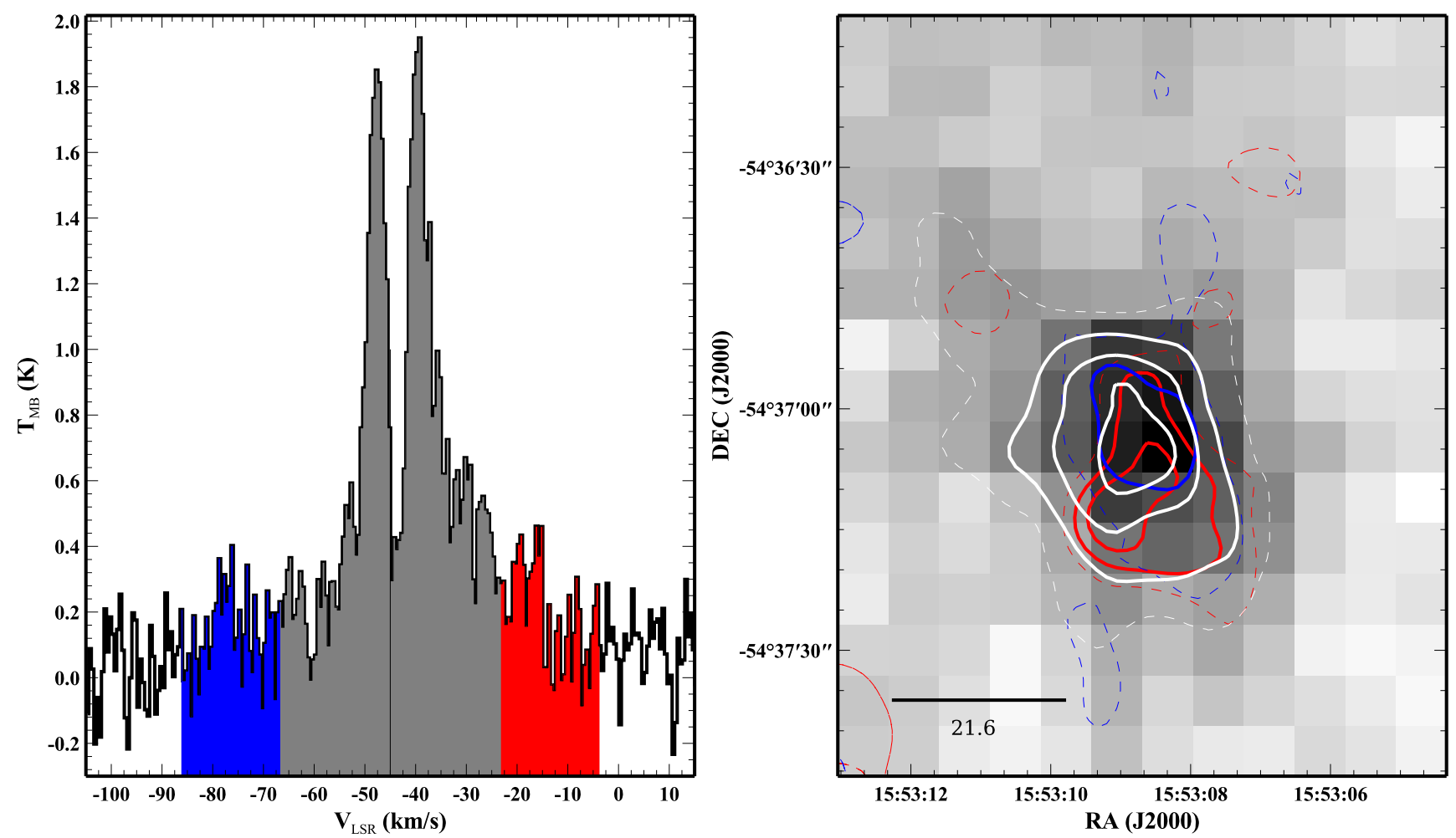

Fig. A.10. As previous figure, for G327. 

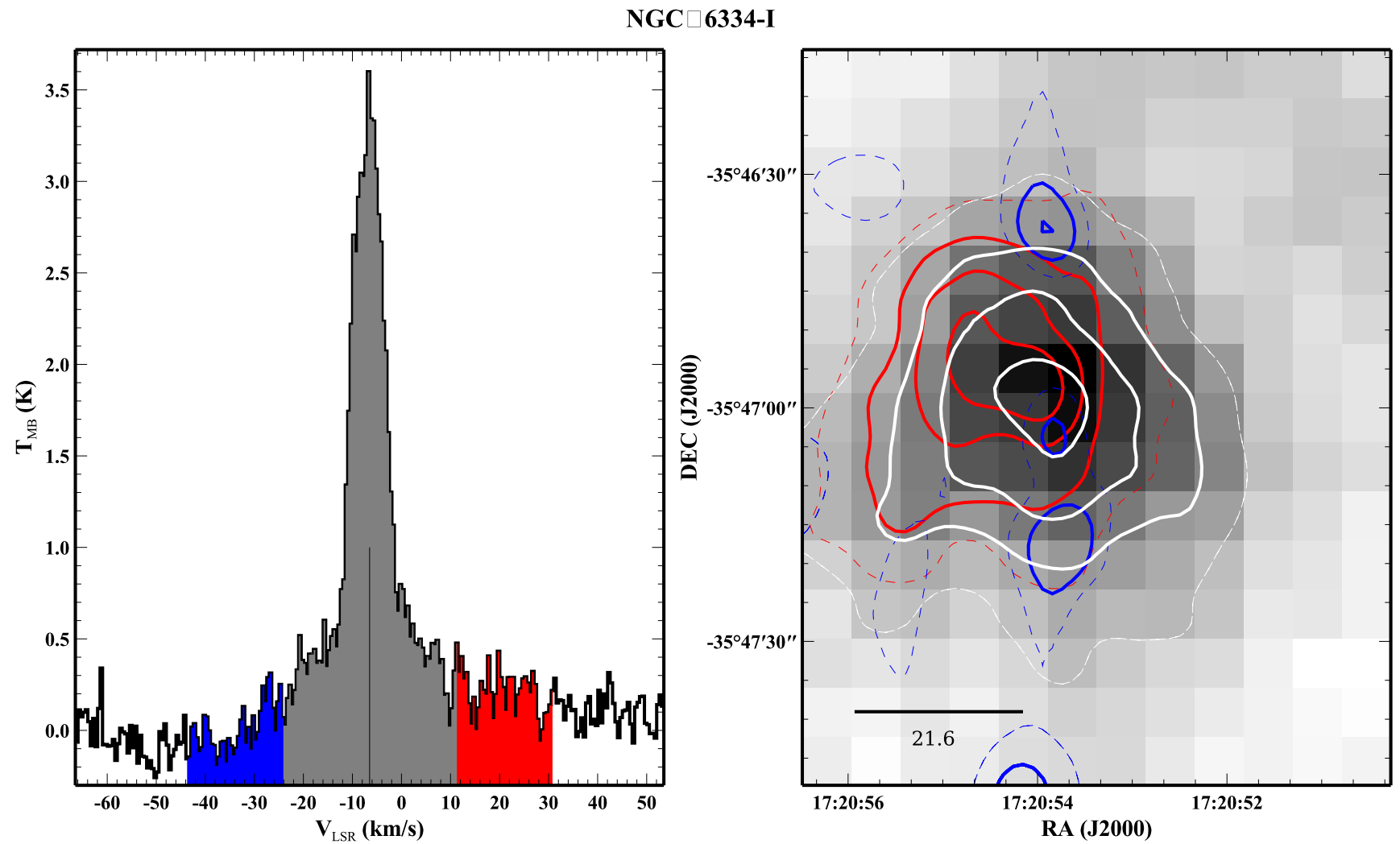

Fig. A.11. As previous figure, for NGC 6334I.
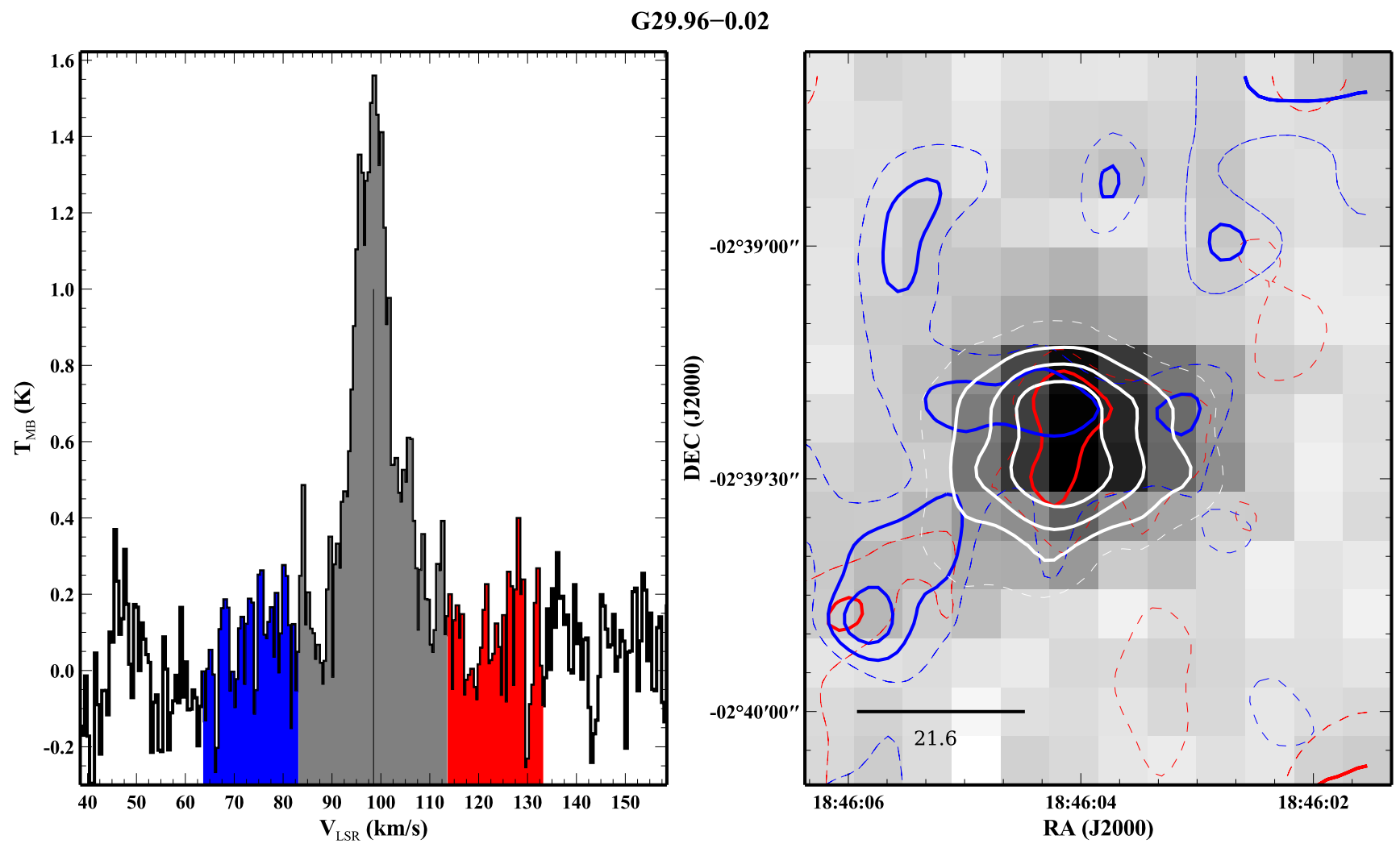

Fig. A.12. As previous figure, for G29.96. 
F. F. S. van der Tak et al.: Infall onto high-mass protostars

G31.41+0.31
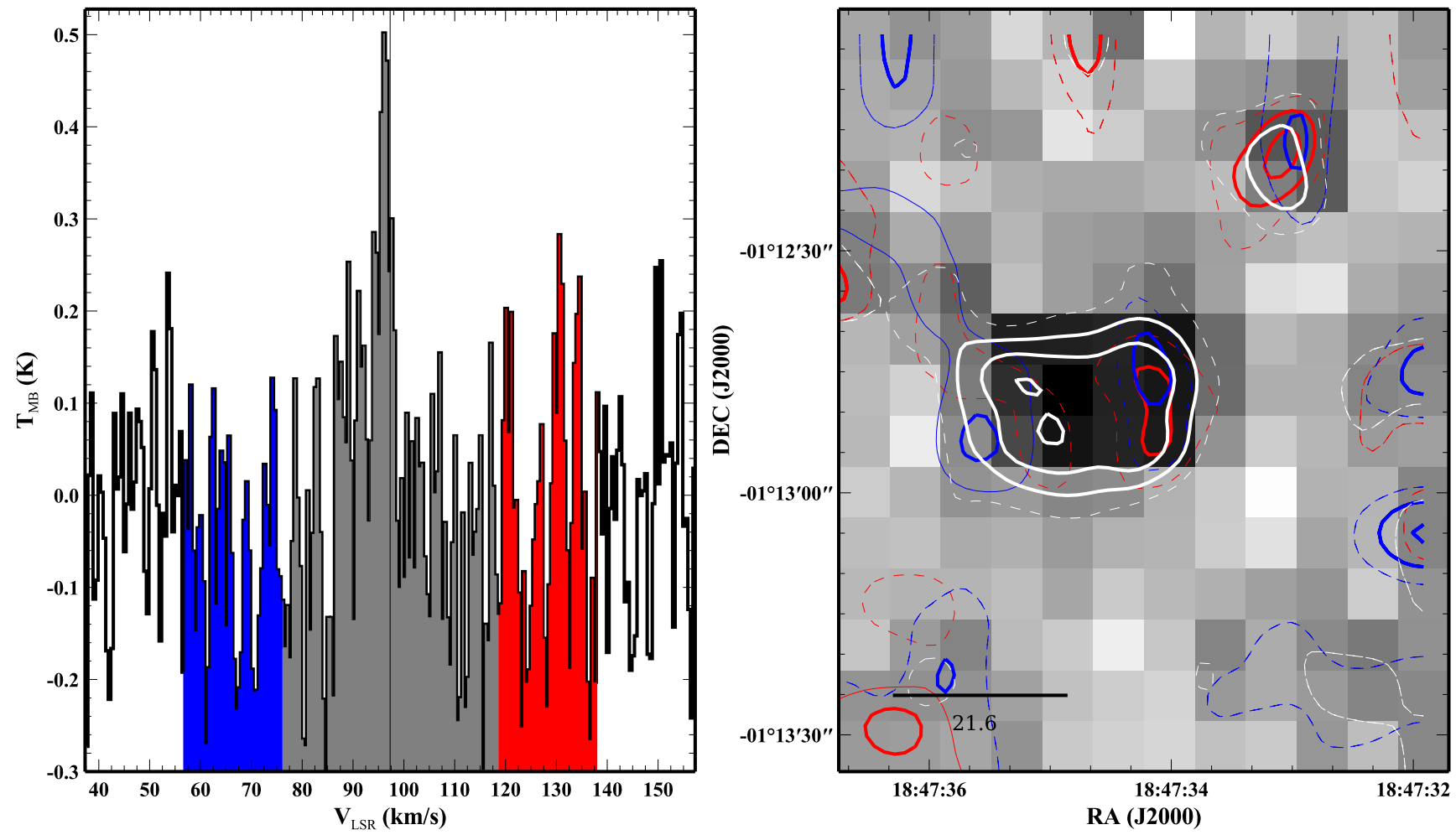

Fig. A.13. As previous figure, for G31.41.

G5.89-0.39
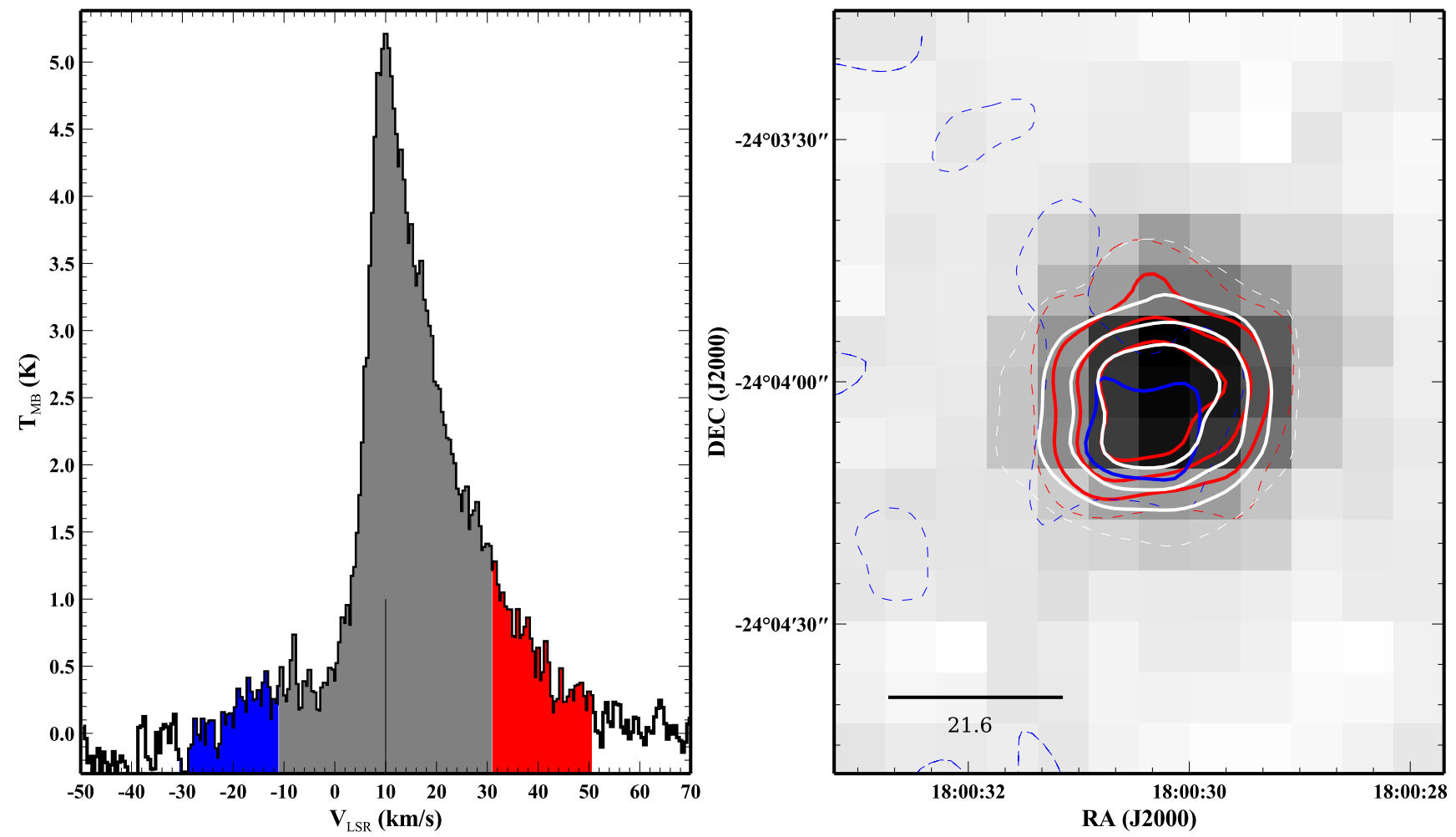

Fig. A.14. As previous figure, for G5.89. 


\section{G10.47+0.03}
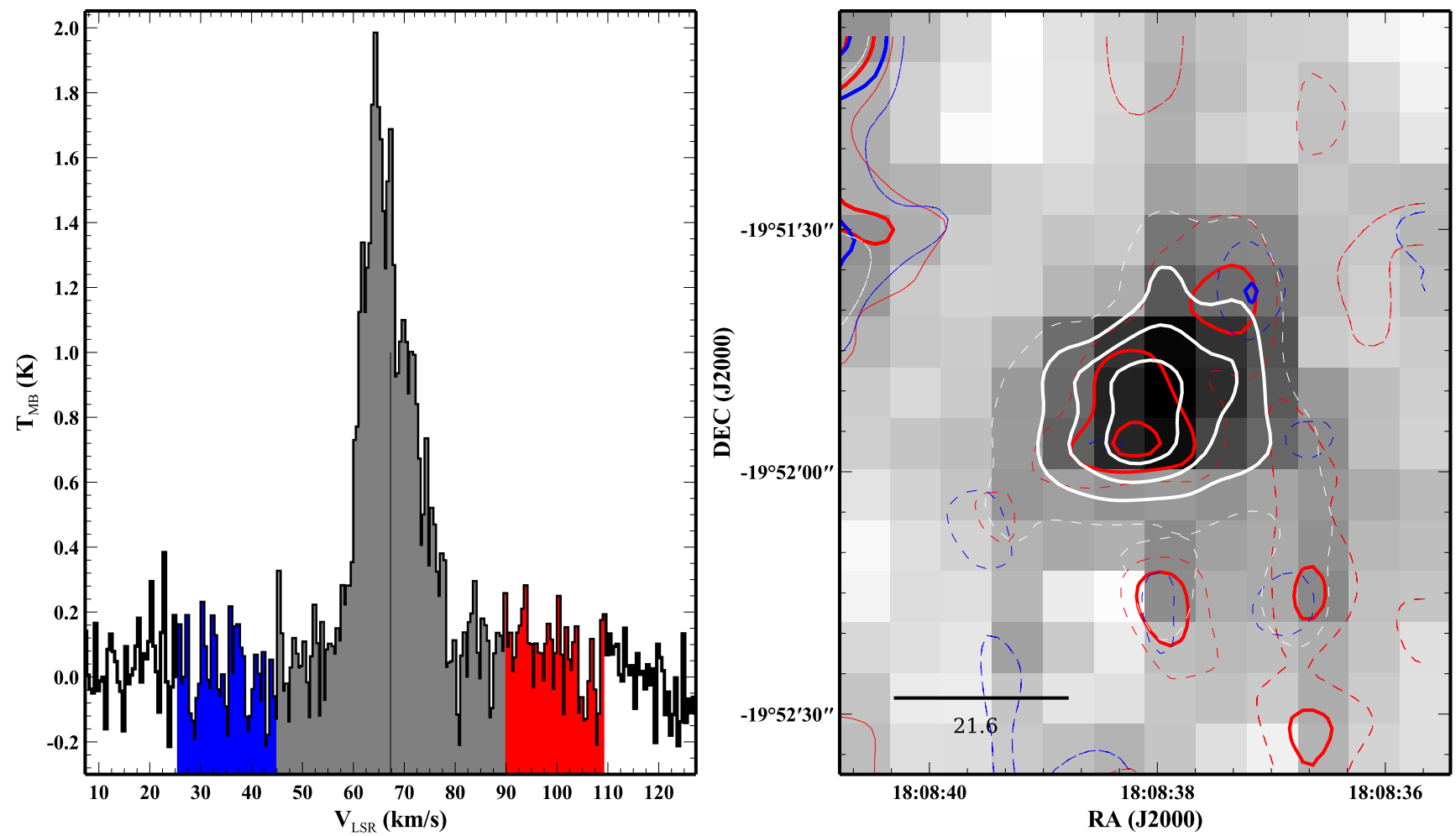

Fig. A.15. As previous figure, for G10.47.

G34.26+0.15
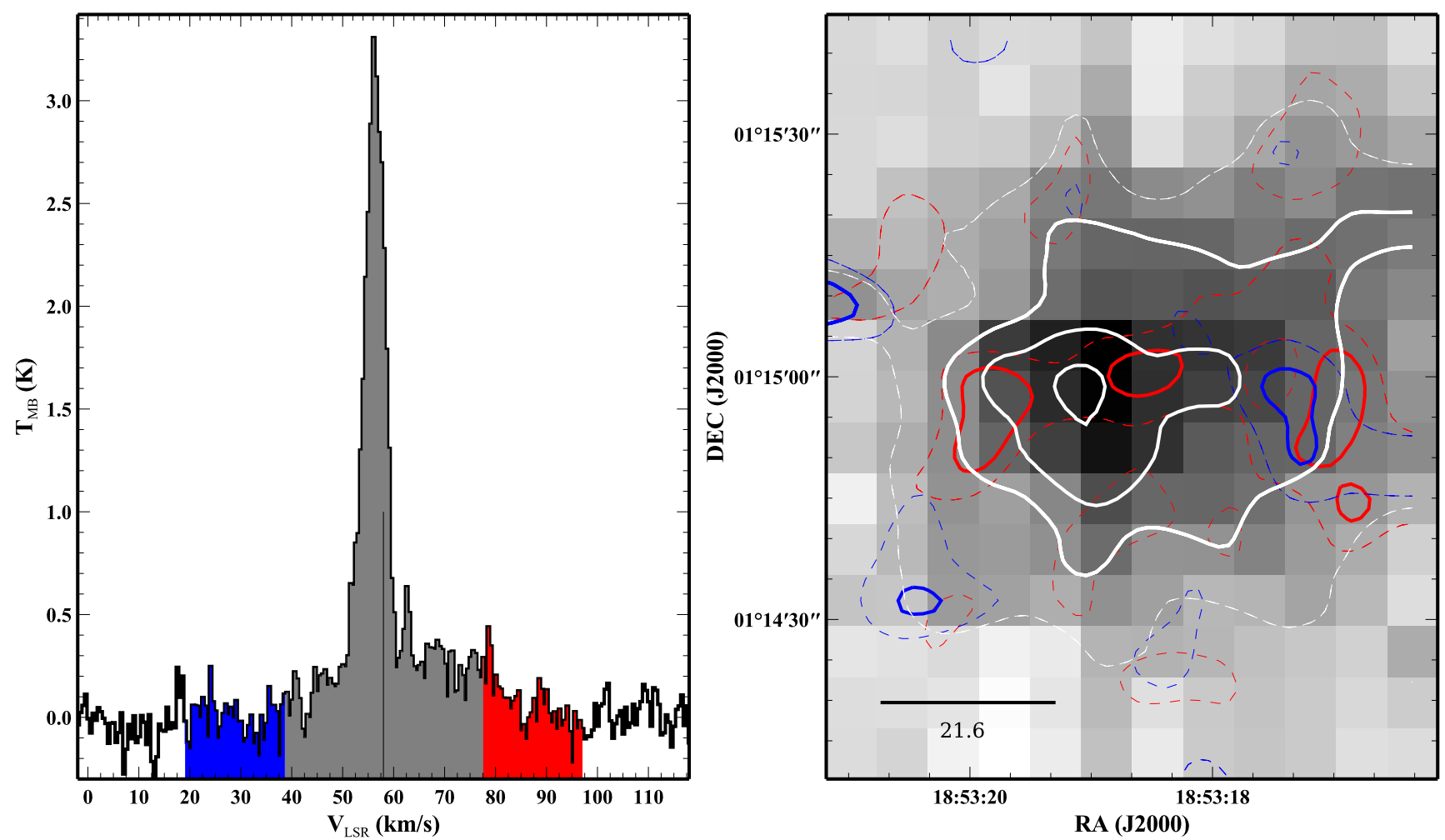

Fig. A.16. As previous figure, for G34.26. 
F. F. S. van der Tak et al.: Infall onto high-mass protostars

W51N-e1
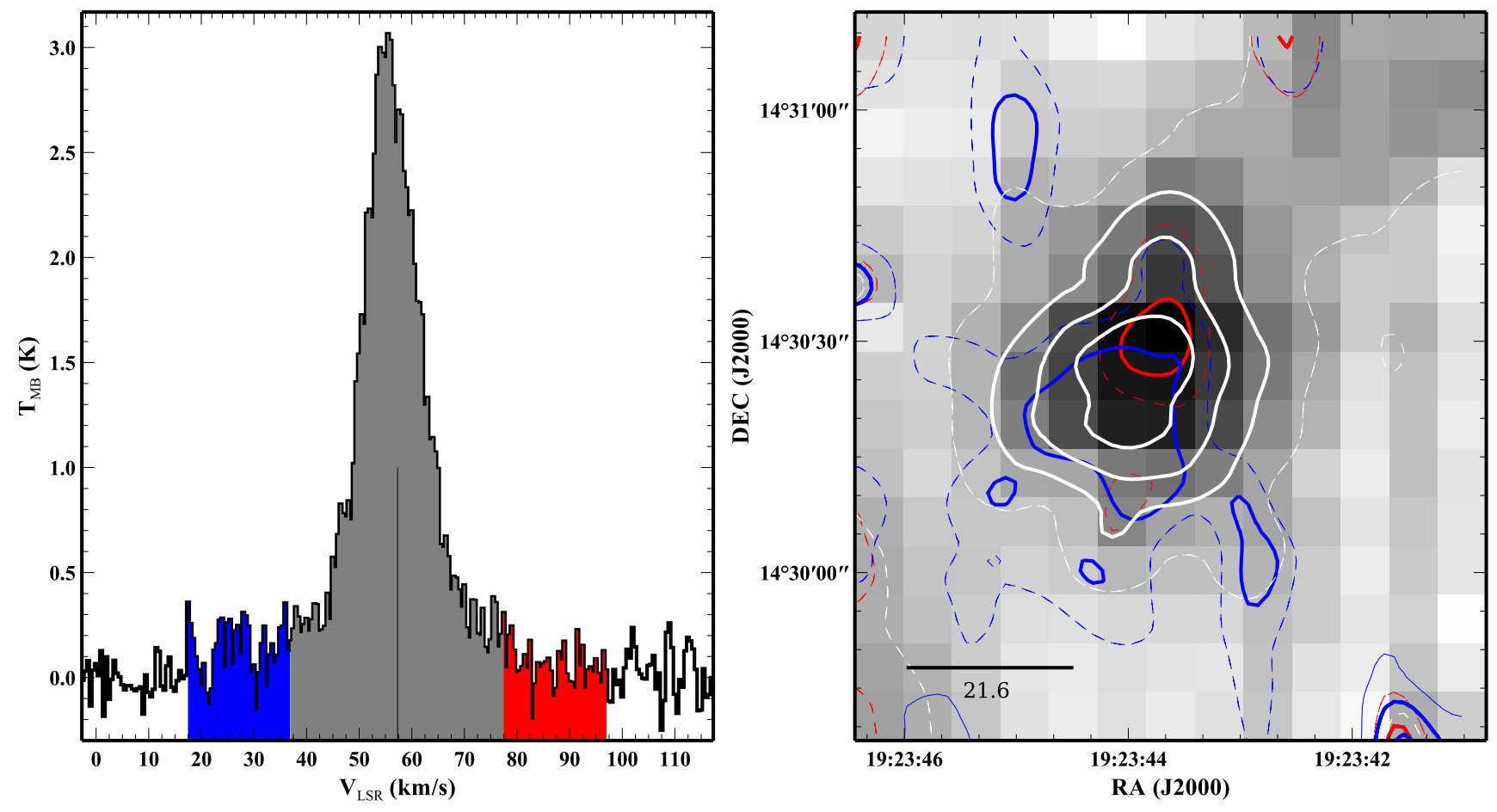

Fig. A.17. As previous figure, for W51N. 